(1)

\title{
土木工程材料自愈合行为的若干力学问题与研究进展
}

\author{
朱兴- ${ }^{1}$, 鲁乘鸿 ${ }^{1}$, 戴子薇 ${ }^{1}$, 李峰 $^{2 *}$ \\ 1. 同济大学道路与交通工程教育部重点实验室, 上海 201804; \\ 2. 北京航空航天大学交通科学与工程学院, 北京 102206 \\ *联系人, E-mail: lifeng98@buaa.edu.cn
}

2020-08-28 收稿, 2020-12-02 修回, 2020-12-04 接受, 2020-12-07 网络版发表

国家自然科学基金 $(51922079,61911530160)$ 和上海市浦江人才计划资助

\begin{abstract}
摘要 为适应未来基础设施和工业化建造需求, 如何改变传统土木工程结构和材料组成, 打造全新的具有智能能 力的基础设施, 已经成为新的研究热点. 工程结构的抗损坏能力直接影响国家的社会成本和经济效益. 为了减少维 修养护费用、提升结构的服役寿命, 一种可行的方案是建造能够进行损伤自我愈合的拟生命系统. 近几年来, 微胶 囊、电沉积、感应加热、微生物自愈合等技术被应用于土木工程与道路工程中，有望提升工程结构的耐久性及稳 定性, 延长服役寿命. 但是, 为提升自愈合工程材料的使用性能、精准预估裂纹扩展轨迹、精确预测材料的使用寿 命, 需要进一步从机理上解释自愈合行为. 本文首先总结了自愈合材料在土木工程中应用的发展历程及研究进展, 随后从损伤力学和断裂力学角度出发, 分析了在解释和预测自愈合行为时所面临的若千力学问题, 并对现有考虑 自愈合效应的本构模型及数值算法进行了梳理. 为了进一步明确各内外因素对裂纹扩展-愈合的正负耦合效应, 从 力学角度提出了叒待解决的问题与挑战.
\end{abstract}

关键词自愈合, 本构模型, 连续损伤自愈合模型, 多尺度, 数值模拟

自愈合材料被广泛应用于土木工程与道路工程中. 当工程材料内部出现裂纹与局部损伤时, 自愈合材料 能进行损伤的自修复和裂纹的自愈合. 自愈合材料在 土木与道路基础设施中的应用有助于提升工程结构的 耐久性及稳定性，延长服役寿命. 《创新 2050: 科技革 命与中国的未来》系列报告提出以先进材料与智能绿 色制造等作为现代化强国经济基础设施主体. 欧盟第 七研发框架计划(FP7)也特别强调加速新兴自愈合材料 的商业化应用. 本文以自愈合土木工程材料赋能新基 建为切人点, 介绍自愈合材料在土木与道路工程中应 用的发展历程和重要进展, 以及在研究自愈合材料自 愈合机理、自愈合机制、服役寿命准确预测等问题中 面临的若干力学问题, 并对其未来发展及应用进行
探讨.

\section{1 土木工程材料的自愈合现象}

在土木工程领域, 多数工程材料具备一定的自愈 合性能, 即在材料服役过程中, 由荷载或环境因素导致 的微损伤和微裂纹能够在一定程度上被自我愈合. 然 而, 材料自身的自愈合效率相对较低. 随着工程技术的 发展, 土木工程领域对建筑材料耐久性和稳定性的要 求越来越高, 仅仅依靠其自身的自愈合, 很难满足要 求. 因此, 向传统工程材料中加人自愈合材料, 通过能 量补给、物质补充等方式激励材料的损伤修复或裂纹 愈合, 从而增强材料的自愈合能力, 这种技术被称为自 愈合能力增强技术. 土木工程材料众多, 本文主要针对

引用格式: 朱兴一, 鲁乘鸿, 戴子薇, 等. 土木工程材料自愈合行为的若干力学问题与研究进展. 科学通报, 2021, 66: 2802-2819 Zhu X Y, Lu C H, Dai Z W, et al. Application of self-healing engineering materials: Mechanical problems and research progress (in Chinese). Chin Sci Bull, 2021, 66: 2802-2819, doi: 10.1360/TB-2020-1082 
土木工程领域中最为常用的材料 水泥基材料和沥 青基材料进行详细介绍.

水泥混凝土的自愈合现象最早于 1836 年提出 ${ }^{[1]}$, 研 究者认为, 这种自愈合能力与混凝土中微溶于水的 $\mathrm{Ca}(\mathrm{OH})_{2}$ 碳化相关. 然而, 也有学者认为, 水泥的自愈合 是通过未水化的水泥颗粒 (如 $\mathrm{C}_{2} \mathrm{~S} 、 \mathrm{C}_{3} \mathrm{~S}$ 等)水化生成水 化产物实现裂纹修复的. Ramm和Biscoping ${ }^{[2] 、 Z h a n g ~}$ 等人 ${ }^{[3]}$ 总结了关于水泥混凝土自愈合现象可能的机理: (1) 未水化的水泥在后期发生水化反应; (2) 裂纹两侧的 混凝土膨胀; (3) 碳酸钻 $\left(\mathrm{CaCO}_{3}\right)$ 结晶; (4) 水中的固体杂 质以及剥落开裂的松散混凝土颗粒填充裂纹. 另一个 重要的道路工程材料——沥青混合料也被证明具有内 在的自愈合能力 ${ }^{[4,5]}$. 通过试验分析及观测发现, 当停止 施加荷载后，沥青混合料的断裂发展区(微、细裂纹聚 集区)将发生应力松驰现象，使得裂纹间隙逐渐缩小. 此时，在分子间的范德华力、氢键的吸附力、毛细流 动作用下，沥青分子将自发地在裂纹界面间进行分子 扩散. 当间歇时间足够长时, 裂纹界面将被沥青分子完 全浸润, 并通过沥青分子的重新组合排列, 使得在无任 何外界作用下, 裂纹逐渐闭合直至消失, 从而恢复沥青 混合料原本的承载能力 ${ }^{[6-8]}$.

虽然这两类材料都具备一定的自愈合性能，但是 由于材料本身的自愈合效率较低，其自愈合程度不能 满足实际工程的需要, 因此, 需要采取自愈合能力增强 技术对工程材料的自愈合性能进行改善，提升材料的 耐久性及稳定性，延长服役寿命. 依据在后期服役期间 是否需要人工干预，可以将自愈合能力增强技术划分 为两大类. 其中, 第一类自愈合能力增强技术只需要在 前期生产过程中掺加特定的材料，在后期服役期间不 再需要人为干预即可提升材料的自愈合性能，如向水 泥混凝土中掺人纤维、氧化镁 $(\mathrm{MgO})$ 、活性外加剂、 高吸水性聚合物等材料, 向沥青中掺人增加沥青强度 或黏度的聚合物或纳米材料等，都能实现较好的增强 效果. 除此之外, 微胶囊、中空纤维、空玻璃管以及微 生物诱导碳酸钻沉淀等也是常见的第一类自愈合能力 增强技术．第二类自愈合能力增强技术则需要在后期 服役期间，通过人为干预以能量或材料补充的形式激 励材料完成自愈合行为. 对于水泥基材料, 主要有电沉 积自愈合增强技术、形状记忆合金(shape memory alloy, SMA)自愈合增强技术、光修复自愈合增强技术以 及通过盐溶液养护加速微裂缝区沉淀以增强自愈合能 力. 对于沥青混合料, 还可以进行加热, 即通过能量供
给来激励损伤愈合.

在水泥混凝土的前期生产中掺人纤维是最为常见 的第一类自愈合能力增强技术，该技术通过纤维提供 的桥联效应，大量结晶产物附着在裂纹内部的纤维上, 逐渐填充裂纹，从而增强混凝土自愈合能力 ${ }^{[9,10]}$. 常用 的纤维有玻璃纤维、金属钢纤维、天然植物纤维和聚 合物有机纤维 ${ }^{[11]}$. 向水泥混凝土中加人氧化美膨胀剂 ( $\mathrm{MgO}$ expansive agent, $\mathrm{MEA}), \mathrm{MgO}$ 发生水化反应, 产 生的 $\mathrm{Mg}(\mathrm{OH})_{2}$ 晶体吸附在 $\mathrm{MgO}$ 颗粒表面及毛细孔壁上, 挤压周围水泥浆体实现裂纹的闭合 ${ }^{[12]}$. 除此之外, 还有 学者提出掺人活性外加剂的渗透结晶自愈合能力增强 技术. 活性外加剂遇水成为一种催化剂, 促进水泥中游 离的 $\mathrm{Ca}^{2+}$ 与 $\mathrm{SiO}_{3}{ }^{2-} 、 \mathrm{AlO}_{3}{ }^{3-}$ 发生络合及结晶沉淀反应, 形成稳定的化合物填充裂纹，实现水泥混凝土的自愈 合 ${ }^{[13 \sim 14]}$. Snoeck等人 ${ }^{[15 ~ 17]}$ 提出, 向混凝土中加人高吸水 性聚合物(superabsorbent polymers, SAP), SAP在水溶液 中可以膨胀到自身重量的数百倍，利用这种膨胀作用 可以对裂纹进行物理堵塞 ${ }^{[18,19]}$, 并且SAP吸收的水分在 后期自愈合过程中可以释放到胶凝基质中，进一步促 进碳酸钙结晶和水化作用 ${ }^{[17,20,21]}$. 对于沥青基材料, 可 以向沥青中掺加能增加其强度或黍度的聚合物，如研 究表明, 苯乙烯-丁二烯-苯乙烯嵌段共聚物(styrene-butadiene-styrene，SBS)改性沥青对沥青混合料的自愈合 能力有提升作用 ${ }^{[22]}$; 也可以向沥青中添加适量的乙烯 基双硬脂酰胺 ${ }^{[23]}$ (ethylene bisstearamide wax，EBS)或 纳米材料(如碳纳米管或纳米黏土 ${ }^{[24 ~ 26]}$ 来增强其自愈 合能力. 此外, 优化沥青混凝土组成设计也可以在一定 程度上增强沥青混凝土自然愈合的能力 ${ }^{[27 ~ 29]}$.

加人微胶囊、中空纤维、中空玻璃管等 ${ }^{[30]}$, 利用 微裂纹打开胶囊，并通过裂纹通道形成的毛细作用和 扩散作用输送包芯材料中的愈合剂，实现裂纹的修补， 从而增强材料的自愈合能力. Dry ${ }^{[31]}$ 于 20 世纪 90 年代提 出, 在水泥混凝土中加人玻璃空心纤维, 其空心纤维内 注人缩醛高分子溶液作为胶黏剂，实现了混凝土的自 愈合. Van Tittelboom等人 ${ }^{[32]}$ 研究了含有胶和剂的中空 纤维在混凝土自愈合方面的应用，并将此技术应用于 实际工程，通过将聚氨基甲酸乙酯包封在中空玻璃纤 维管内后放置于混凝土梁中, 实现了裂纹的自动愈合, 降低了钢筋锈蚀的风险. 2010年，García等人 ${ }^{[33]}$ 首次将 微胶囊技术正式应用于沥青自愈合，并以环氧树脂和 水泥为胶囊壁, 包裹吸收了再生剂的多孔砂, 制备了沥 青自愈合微胶囊. 随后, 更多的学者展开了微胶囊技术 
在沥青自愈合方面的研究, 其中Sun等人 ${ }^{[34]}$ 制备了三聚 氰胺艮甲醛微胶囊, 结合光学和扫描电子显微镜以及 动态剪切流变仪(dynamic shear rheometer, DSR)试验 等, 对不同微胶囊含量的沥青愈合效率进行了评价.

微生物诱导碳酸钲沉淀增强材料自愈合能力的原 理主要分为两类. 一类是将新拌混凝土与特殊微生物 (如好氧嗜碱芽狍杆菌)直接混合, 这种微生物在高碱、 缺氧条件下处于休眠状态, 当混凝土表面出现裂纹, 氧 气和水分的渗人唤醒休眠的细菌孢子, 细菌新陈代谢产 生的 $\mathrm{CO}_{2}$ 与 $\mathrm{Ca}^{2+}$ 发生反应生成碳酸钙沉淀, 填补裂 纹 ${ }^{[35 ~ 37]}$. 另一类是考虑到混凝土较高 $\mathrm{pH}$ 、相对干燥的 环境不适宜细菌的存活, 将细菌狍子包裹在水凝胶、 硅胶、玻璃毛细管中, 而后置于混凝土中, 待裂纹出现 导致细菌孢子外封装破裂, 进而完成后续的微生物诱导 碳酸钙沉淀实现自愈合 ${ }^{[38-40]}$. 除此之外, 还有学者将微 生物诱导自愈合技术与纤维增强技术进行融合, 提出用 天然纤维或人工纤维包裹细菌狍子放置于水泥砂浆中, 纤维通过在裂纹上起桥梁作用, 增加了裂纹区域细菌的 可利用性, 进一步提高了砂浆的自愈合能力 ${ }^{[41]}$.

在第二类自愈合能力增强技术中，电沉积自愈合 能力增强技术是将带裂纹的混凝土放置在电解液中, 通过外加阳极形成闭合回路, 利用电场作用下电解液 发生化学反应生成沉淀, 填充裂缝进而实现自愈 合 ${ }^{[42-45]}$. 形状记忆合金自愈合能力增强技术是通过将 预拉伸的SMA埋人混凝土拉伸区，当裂缝超过最大宽 度限制时, 对附近的SMA加热, 激励其收缩变形, 从而 实现裂缝的收缩和愈合 ${ }^{[46 ~ 49]}$. 光修复自愈合能力增强 技术的愈合机制是在紫外光的刺激下，愈合剂分子由 基态转化为激发态产生自由基，并进行重排反应和成 对键, 从而实现裂缝的修复 ${ }^{[50]}$. 盐溶液养护通过加速微 裂缝区沉淀物(如C-S-H凝胶等)的生成, 从而缩短修复
时间 ${ }^{[51]}$, 增强自愈合能力. 1967年, Bazin和Saunie ${ }^{[52]}$ 提 出一种思想, 认为提高温度能加快沥青分子的扩散速 率, 基于这个思想, 出现了对沥青混合料进行加热以提 供能量的自愈合能力增强技术. 目前, 能量供给的沥青 混合料自愈合能力增强技术主要有 3 个方面, 包括导电 加热、微波加热和磁感应加热，如在沥青混合料中掺 加导电纤维、石墨等具有导电能力的材料 ${ }^{[53,54]}$, 或掺 人钢纤维、钢丝线等具有磁感应功能的材料 ${ }^{[55 ~ 59]}$, 或 加人铁氧体等能够提升微波吸热的材料 ${ }^{[60,61]}$, 分别通 过对导电材料通电、电磁感应加热、释放微波的方法 使沥青路面产生热量, 促进沥青材料自愈合.

\section{2 土木工程材料自愈合行为的力学问题}

在整个服役期内，自愈合土木工程材料在受到疲 劳破坏的同时也经历着不断的自我修复，其实际损伤 破坏过程非常复杂. 在一定服役期后, 材料内部会同时 产生损伤及可见裂纹. 由于自愈合材料的加人(如微胶 囊等)以及材料自身可能存在的自愈合能力, 损伤和可 见裂纹均会部分愈合. 随后, 在荷载的继续作用下, 部 分愈合的损伤会进一步演化，而部分愈合的可见裂纹 会进一步扩展. 循环往复, 直至材料的疲劳破坏.

从损伤力学及断裂力学的角度来看, 裂纹往往是 在受损伤的材料中发展起来，而在宏观裂纹形成之后， 细观损伤仍在不断演化, 并推动宏观裂纹的发展, 如图 1(a) (c). 而对于自愈合复合材料, 自愈合在整个过程中 也起到潜移默化的关键作用. 因此, 若要对自愈合材料 的损伤-断裂-愈合过程进行精细化分析, 则应同时考虑 损伤、断裂、自愈三者的共同作用, 如图1(d)所示.

此外，土木工程材料中普遍存在的黏弹塑性使得 损伤-自愈机制更为复杂. 黏弹性材料会表现出触变 性、弹性恢复等现象. 这些现象从表象上来说, 都会对

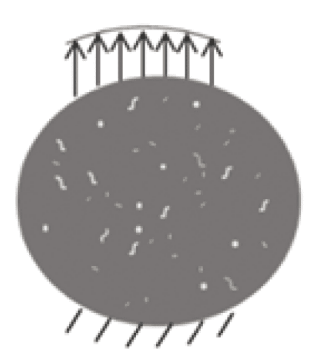

(a)

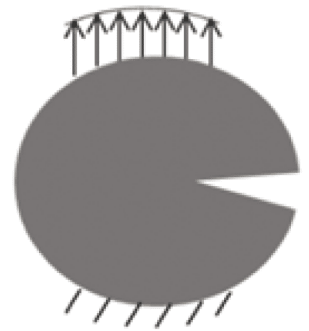

(b)

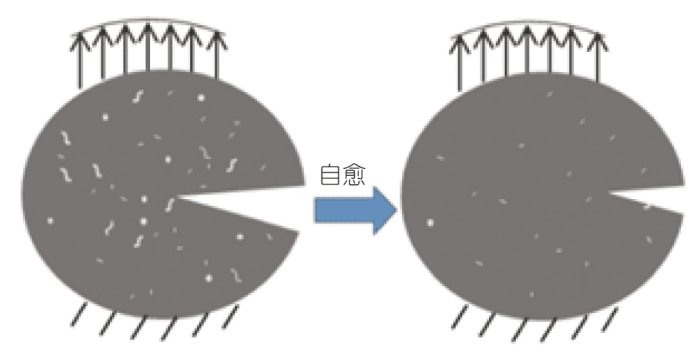

(d)

图 1 (网络版彩色)力学模型. (a) 经典损伤力学模型; (b) 经典断裂力学模型; (c) 同时考虑损伤及断裂模型; (d) 同时考虑损伤、断裂、自愈模型 Figure 1 (Color online) Mechanical models. (a) Classical damage mechanical model; (b) classical fracture mechanical model; (c) damage-fracture model; (d) damage-fracture-self-healing model 
材料的性能恢复产生积极贡献，但其本质上与自愈合 又完全不同. 黏弹性本身的性能恢复一般发生在间歇 期(撤去荷载时), 并且不一定与损伤断裂相关. 而自愈 合不仅出现在间歇期，在损伤累积时也同样可能会出 现. 两者尽管有本质上的区别, 但归根结底, 对宏观性 能的恢复都有正效应. 如何将这两种效应解耦也是一 大难点. 尤其对于黏弹塑性材料而言, 在考察其当前的 应变响应时, 以前的加载历史也会对以后的变形产生 影响. 而且, 黏弹塑性体的力学响应与加载速率和时间 密切相关, 还会受到温度的影响. 通常情况下, 随着温 度的升高, 黏弹塑性材料的力学响应越来越明显地表 现出黏性流体的特征; 而随着温度的降低, 其弹塑性固 体的特征越来越明显. 除此之外, 黏弹塑性体的受荷响 应还与加载方式、加载历史等因素密切相关.

\section{3 考虑自愈合效应的本构模型}

无论是自然愈合还是自愈合能力增强技术，均是 基于材料自身具有自我修复的固有属性. 从力学角度 建立考虑自愈合效应的本构模型，可以帮助我们进一 步研究分析材料的自愈合机理.

目前，关于损伤-自愈耦合效应的理论研究大都建 立在连续损伤力学的基础之上. 2005年，Barbero等 人 $^{[62]}$ 对连续损伤力学进一步推广, 首次提出连续损伤自愈模型(continuum damage-healing mechanics, $\mathrm{CDHM}$ ), 并给出了弹塑性损伤-自愈的应力应变曲线 (图2), 用以研究聚合物复合材料的自愈合行为. 其假 定损伤和愈合随着应力的增加而增加, 在一个加载循 环过程中, 消耗的总能量以 $\overline{O A B C E}$ 的面积表示, 愈合效

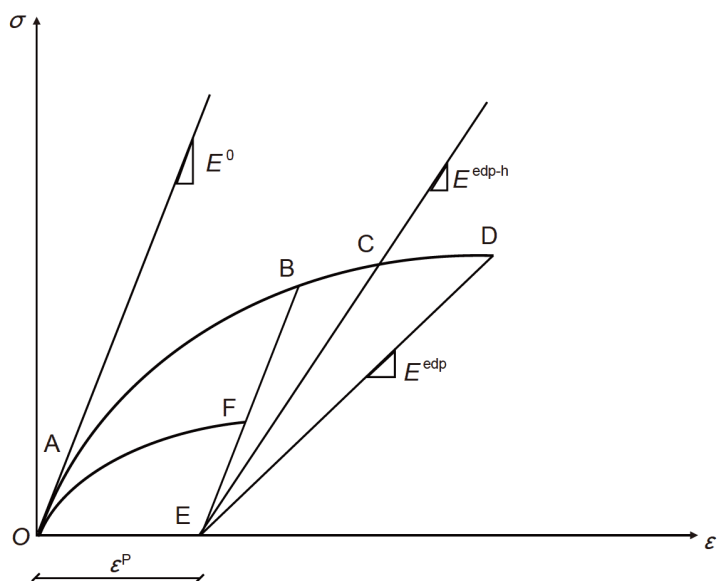

图 2 弹塑性损伤-自愈的应力应变曲线

Figure 2 Stress-strain curve: Elasto-plastic-damage-healing
应带来的恢复能和损伤效应及塑性引起的耗散能分别 用 $\overline{C D E} 、 \overline{B D E}$ 及 $\overline{O A F E}$ 的面积表示. 自愈合现象是由内 能产生而引发的, 内能可以通过消耗储存在愈合剂中 的化学能获得, 也可以通过能量补给和物质补充获得. 在单轴应力状态下，损伤能量和愈合能量分别沿 着 $\overline{B C D}$ 和 $\bar{C}$ 路径耗散.

依据连续损伤力学中的概念, 材料劣化的主要机 制是由微损伤导致的有效承载面积的减小，那么材料 的自愈可视为有效承载面积的增加. 基于上述概念, 名 义应力张量 $\boldsymbol{\sigma}$ 和有效应力张量 $\boldsymbol{\sigma}$ 的关系式如式(1):

$\boldsymbol{\sigma}=[1-D(1-h)] \overline{\boldsymbol{\sigma}}$,

其中, 内变量 $D$ 为损伤变量, 以表征材料强度的退化 $(D \in[0,1])$; 内变量 $h$ 为自愈合变量, 以表征材料强度的 恢复 $(h \in[0,1])$. 通过式(1), 可将损伤、自愈合效应体现 在材料的本构模型中. 而常见的连续损伤-愈合模型建 立在热力学框架的基础之上，因此，需要对损伤愈合构 型和有效构型(图3)中的应变进行一定的假设 ${ }^{[63]}$, 以将 有效构型和损伤愈合构型建立起联系. 通常采用的假设 有应变等效假设、应变能等效假设、功率等效假设.

随着荷载的继续作用, 部分损伤会进一步演化, 形 成宏观裂纹，此时仅依靠材料的自然愈合很难使其恢 复到正常工作状态. Barenblatt ${ }^{[64]}$ 和Dugdale ${ }^{[65]}$ 在连续损 伤力学的基础上, 提出了用于断裂力学的内聚力模型 (cohesive zone model, CZM), 裂纹扩展过程被视为内 聚区材料连续损伤至断裂的过程. 裂纹的形成可以分 为 4 个阶段, 分别是微损伤的出现, 微损伤处即将 (最 早)出现局部变形，裂纹的演化和发展以及裂纹出现、 自由表面形成. 其中, 阶段 2 需要被单独考虑, 此时区域 处于过渡阶段，该区域又被称作裂纹尖端附近的塑性 屈服区(内聚区), 材料的行为发生显著变化. 建立该区 域的汼引-分离准则, 可以帮助预测微损伤是否具有发 展成裂纹的趋势 ${ }^{[66]}$. 之后, 通过在内聚力模型中引人愈 合变量, 可以很好地诠释裂纹的开裂-愈合过程. 除此之 外, 内聚力模型能够将微观损伤与宏观断裂进行衔接, 建立起损伤力学与断裂力学之间的联系.

\section{1 基于应变等效假设的CDHM模型}

应变等效假设是在构造受损材料本构方程时最常 用的假设, 它使得本构模型的理论推导和数值实现变 得相对简单. 但是, 在大变形或较大损伤演化的情况 下, 一般不宜使用该假设. 


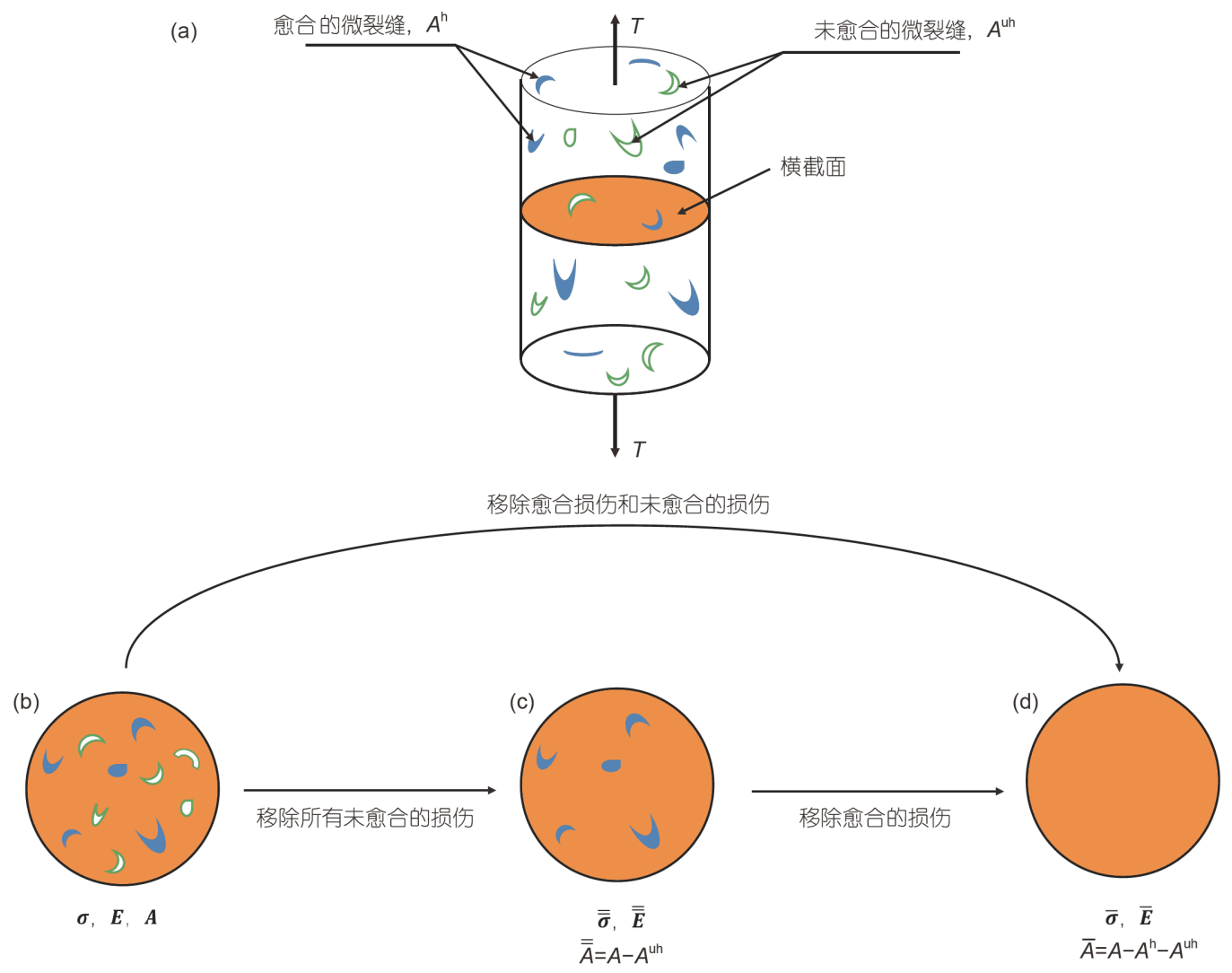

图 3 (网络版彩色)连续损伤-自愈模型基本概念. (a) 损伤自愈构型; (b) 名义构型; (c) 自愈构型; (d) 等效构型

Figure 3 (Color online) Continuum damage-healing configuration. (a) Damage-healing configuration; (b) norminal configuration; (c) healing configuration; (d) effective configuration

Abu Al-Rub等人 ${ }^{[67]}$ 分别采用Schapery的非线性黏弹性 理论和Perzyna的黏塑性本构关系来求解沥青混合料的 黏弹性和黏塑性响应. 基于应变等效假设, 将应变分为 黏弹性应变和黏塑性应变, 应变等效假设可表示为

$$
\varepsilon_{i j}=\bar{\varepsilon}_{i j}, \varepsilon_{i j}^{\mathrm{ve}}=\bar{\varepsilon}_{i j}^{\mathrm{ve}}, \varepsilon_{i j}^{\mathrm{vp}}=\bar{\varepsilon}_{i j}^{\mathrm{vp}},
$$

其中, $\varepsilon, \varepsilon^{\mathrm{ve}}, \varepsilon^{\mathrm{vp}}$ 分别是总应变张量、黏弹性应变张量和 黏塑性应变张量; $\bar{\varepsilon}, \bar{\varepsilon}^{\mathrm{ve}}, \bar{\varepsilon}^{\mathrm{vp}}$ 分别是有效总应变张量、 有效黏弹性应变张量和有效黏塑性应变张量.

在黏性损伤求解部分，采用以损伤变量表示的考 虑时间和温度效应的损伤演化方程:

$$
\begin{aligned}
\dot{D}= & \Gamma_{0}^{\mathrm{vd}}\left(\frac{\bar{Y}}{Y_{0}}\right)^{q}[1-D(1-h)]^{2} \\
& \times \exp (k \bar{\varepsilon}) \exp \left[-\alpha_{1}\left(1-\frac{T}{T_{0}}\right)\right],
\end{aligned}
$$

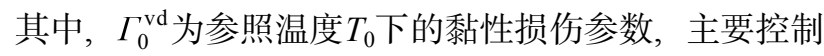
损伤发生速率; $Y_{0}$ 为参照温度下的损伤力; $Y$ 为用有效 应力表示的损伤驱动力; $\overline{\boldsymbol{\varepsilon}}$ 为有效应变; $q$ 为应力依赖参
数; $k$ 和 $\alpha_{1}$ 为相关材料参数, 主要基于试验来确定.

参考黏性损伤部分的求解，建立同样考虑时间和 温度效应的愈合演化方程如式(4):

$$
\dot{h}=\Gamma_{0}^{\mathrm{h}}[1-D(1-h)]^{\beta_{1}}(1-h)^{\beta_{2}} \exp \left[-\alpha_{2}\left(1-\frac{T}{T_{0}}\right)\right],
$$

其中, $\Gamma_{0}^{\mathrm{h}}$ 为参照温度 $T_{0}$ 下的黏性愈合参数; $\alpha_{2}, \beta_{1}$ 和 $\beta_{2}$ 为 相关材料参数, 主要由试验确定.

Voyiadjis等人 ${ }^{[68]}$ 基于应变等效假设的CDHM模型, 提出了新的各向异性损伤-愈合变量, 可更为准确地预 测材料的损伤与愈合行为. 他们进一步研究了基于形 状记忆聚合物的自愈合系统的非弹性损伤响应.

\section{2 基于功率等效假设的CDHM模型}

基于功率等效(power equivalence)假设，可建立与 耗散能相关的CDHM模型, 该模型具有更明确的物理 意义且能更为准确地预估耗散能. 此外, 基于该假设, 在构造数值算法时也会相对简单. 名义构型 $(\Pi)$ 和有效 
构型 $(\bar{\Pi})$ 中的功率消耗可表示为

$\Pi=\frac{1}{2} \sigma: \dot{\varepsilon} ; \bar{\Pi}=\frac{1}{2} \bar{\sigma}: \dot{\bar{\varepsilon}}$.

功率等效假设认为, 名义构型中的功率消耗与有效构 型中的功率消耗相同, 即

$\Pi=\bar{\Pi}$.

Darabi等人 ${ }^{[63]}$ 基于上述CDHM模型, 提出了损伤演 化函数和愈合演化函数, 分别如式(7)和(8)所示:

$\dot{\phi}=\Gamma^{\mathrm{vd}}\left(\frac{\bar{Y}}{Y_{\mathrm{th}}}\right)(1-\phi)^{2} \exp \left(k \bar{\varepsilon}_{\mathrm{eff}}\right)$,

$\dot{h}=\Gamma^{\mathrm{h}}(1-\phi)^{m_{1}}(1-h)^{m_{2}}$,

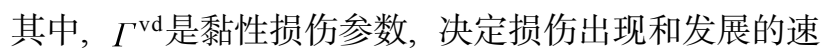
度; $\phi, h$ 是损伤变量和愈合变量; $\bar{\varepsilon}_{\text {eff }}$ 是有效应变; $Y_{\mathrm{th}}$ 是 损伤阈值; $Y$ 是损伤驱动力; $m_{1}, m_{2}$ 是材料参数, 由试验 决定. 在此基础上, 利用功率等效假设得到试验中 $\Delta t$ 时 间内应变张量的增量, 采用迭代方法获得 $t+\Delta t$ 时刻有效 构型中的总应变张量, 并代人胡克定律, 便能得到当前 时刻的应力, 从而计算得到新的损伤和愈合变量.

\section{3 基于应变能等效假设的CDHM模型}

应变能等效假设认为, 名义构型中的应变能与有 效构型中的应变能相等，但在实际应用中，考虑到 CDHM模型的热力学描述通常只适用于小应变、各向 同性材料, 因此应变能等效假设也可简化为弹性能等 效假设.

名义构型 $(W)$ 和有效构型 $(W)$ 中的弹性应变能可表 示为

$W=\frac{1}{2} \sigma: \varepsilon ; W=\frac{1}{2} \bar{\sigma}: \bar{\varepsilon}$.

应变能等效假设则表示为

$W=\bar{W}$.

分别引入标量损伤函数和标量愈合函数，如式(11)和 (12)所示:

$g^{\varphi}\left(y^{\varphi}, s^{\varphi}\right)=\frac{1}{2}\left(y^{\varphi}\right)^{2}-L^{\varphi}\left(s^{\varphi}\right)$,

$g^{h}\left(y^{h}, s^{h}\right)=\frac{1}{2}\left(y^{h}\right)^{2}-L^{h}\left(s^{h}\right)$,

其中, $L^{\varphi}\left(s^{\varphi}\right)$ 表示损伤强化参数, 是全局损伤参数 $s^{\varphi}$ 的 函数; $L^{h}\left(s^{h}\right)$ 表示愈合强化参数; $y^{\varphi}$ 是与损伤变量相对
应的广义热力学力; $y^{h}$ 是与愈合变量相对应的广义热 力学力; $s$ 是全局愈合参数. 引人耗散能函数, 对损伤 愈合的正交法则进行推导，通过拉格朗日乘子和稳定 态条件进行约束, 并应用连续性条件, 结合上述公式进 行数学推导转化, 可最终得到应变-损伤和应变-愈合的 关系.

Esgandani和El-Zein ${ }^{[69]}$ 基于应变能等效假设的 CDHM模型, 引人非饱和土的边界面塑性理论, 建立了 弹塑性-损伤-愈合本构模型, 并在热力学框架内考虑了 塑性硬化、应变率、应力比、吸力硬化和围压的影响, 建立了黏性土损伤和愈合的演化规律. 通过将模拟结 果与已有的实验数据进行比较, 证实了模型的可行性. 对比应变控制条件下排水三轴试验中的仿真结果与试 验数据, 结果表明, 考虑自愈合效应后, 预测结果更接 近试验值. Voyiadjis等人 ${ }^{[70]}$ 基于弹性应变能等效假设 理论的CDHM模型，引人损伤和愈合过程的各向同性 硬化效应，提出了考虑各向同性硬化效应的两种新的 损伤和愈合屈服面，研究了弹塑性损伤-愈合耦合问题 的计算方法, 得到了聚合物自愈合体系的力学行为, 并 对基于形状记忆聚合物的自愈合材料进行了单轴压缩 试验. 结果表明, 所提出的本构方程能够有效地模拟损 伤和愈合过程, 且能准确地模拟基于形状记忆聚合物 的自愈合材料的力学行为. 此外, Voyiadjis等人 ${ }^{[68]}$ 进一 步研究了单轴加载情况下自愈合材料损伤和愈合过程 的力学, 给出了分别基于面积变化和弹性模量变化的 损伤参数表达式 $(\phi, l)$ 和愈合参数表达式 $\left(h, h^{\prime}\right)$, 并分别 利用弹性应变等效假设和弹性应变能等效假设推导了 四者之间的关系, 最后基于热力学连续损伤框架, 建立 了损伤愈合的演化规律，推导了自愈合材料在不同愈 合策略下的数学表达式, 研究了愈合对经典损伤变量 $\phi$ 上界的影响.

\section{4 考虑多种影响因素的损伤自愈修正模型}

Oucif等人 ${ }^{[71]}$ 对Mazars混凝土损伤模型进行扩展, 通过引人愈合变量 $h$, 建立了损伤愈合模型. 该模型描 述了耦合与非耦合自愈合机制下的愈合效率，认为愈 合在变形和非变形情况下均会发生. 损伤历史、间歇 时间和材料特性等参数均会影响愈合效率, 因此, 该愈 合模型的主要特征是考虑了影响愈合效率的参数. 通 过材料参数 $\gamma$ 定义愈合进展的快慢. 结合试验研究, $\mathrm{Ou}-$ $\mathrm{cif}^{\text {等人 }}{ }^{[71]}$ 提出了具有时间依赖性的愈合方程: 


$$
h(t)=1-\exp \left[-\gamma \varphi\left(t_{\mathrm{h}}\right)\left(t_{\mathrm{hf}}-t_{\mathrm{hi}}\right)\right]
$$

其中, $\gamma$ 是材料参数; $\varphi\left(t_{\mathrm{h}}\right)$ 是愈合过程 $t_{\mathrm{h}}$ 时的损伤变量, 需要注意的是, 在卸载阶段 $(\dot{\varphi}=0), \varphi\left(t_{\mathrm{h}}\right)$ 是常数; $t_{\mathrm{hi}}$ 是愈 合开始时间; $t_{\mathrm{hf}}$ 是愈合结束时间; $t_{\mathrm{h}} \in\left[t_{\mathrm{hi}}, t_{\mathrm{hf}}\right]$. 该模型考 虑了加载阶段材料的愈合行为, 即认为损伤和愈合同 时发生. 在这种情况下, $\varphi\left(t_{\mathrm{h}}\right)$ 将会一直演化直至材料失 效 $(\dot{\varphi}>0)$.

Shahsavari等人 ${ }^{[72]}$ 基于CDHM模型对损伤演化方 程和愈合演化方程进行了改进. 损伤演化方程是在Dar$a b i$ 等人 2011 年 ${ }^{[73]}$ 和 2013 年 ${ }^{[74]}$ 分别提出的损伤演化方 程基础上进行的扩展. 他们为损伤力和损伤变量各自 分配一个特定的材料参数，从而更好地预测材料的损 伤行为; 并通过使用损伤饱和函数限制损伤变量最大 不超过单元整体, 最终所采用的损伤演化方程为

$$
\dot{D}=\Gamma^{\mathrm{vd}}\left(\frac{\widetilde{Y}_{\mathrm{eq}}}{Y_{0}}\right)^{X_{1}}(1-\widetilde{D})^{X_{2}} \exp \left(K_{\mathrm{D}} \widetilde{\varepsilon}_{\mathrm{eq}}\right) .
$$

该损伤演化方程主要分为四部分, 第一部分 $\Gamma^{\mathrm{vd} \text { 表示材 }}$ 料参数, 与损伤速率呈线性相关. 第二部分是损伤与等 效有效应力 $\left(\widetilde{Y}_{\mathrm{eq}}\right)$ 之间的关系，其中 $Y_{0}$ 是基准损伤力，与 材料本身的性质有关. 第三部分是损伤饱和函数, 以保 证损伤演化方程符合实际, $\widetilde{D}$ 是有效损伤变量. 第四部 分建立了与有效应变的联系, $\widetilde{\varepsilon}_{\mathrm{eq}}$ 是等效有效应变. 在该 模型中, 损伤增长是等效有效应变的指数函数, $X_{1}$ 、 $X_{2} 、 K_{\mathrm{D}}$ 分别表示应力、损伤和应变灵敏度参数.

愈合演化方程则是基于Voyiadjis等人 ${ }^{[68]} 、 \mathrm{Abu} A 1-$ Rub等人 ${ }^{[67]}$ 、Darabi等人 ${ }^{[63]}$ 的研究, 对损伤和愈合变量 进行解耦, 分别配置两个不同的材料参数, 从而更好地 预测愈合行为. 与损伤饱和函数类似, 也引人愈合饱和 函数以限制愈合变量最大不超过单元整体. 值得注意 的是, 该模型认为, 愈合是从一个特定的损伤值开始增 长, 这在之前的损伤演化方程中是没有提到的. 愈合演 化方程如式(15)所示:

$$
\dot{h}=u\left(\widetilde{D}-D_{0}\right) \Gamma^{\mathrm{h}}(1-D)^{Y_{1}}(1-h)^{Y_{2}},
$$

其中, 第一部分中, $u(\cdot)$ 为Heaviside阶梯函数, $D_{0}$ 是愈合 开始时的损伤阈值; 第二部分 $\Gamma^{\mathrm{h}}$ 作为材料参数, 给定了

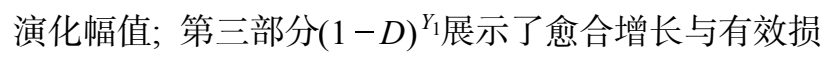
伤之间的关系; 第四部分是损伤饱和函数, 以确保愈合
演化符合实际. $Y_{1}$ 和 $Y_{2}$ 是愈合材料参数; $\widetilde{D}=D(1-h)$, 代表有效损伤.

Alsheghri和Abu Al-Rub ${ }^{[75]}$ 基于热力学定律和虚功 原理，建立了模拟自愈合材料裂纹愈合的内聚力愈合 模型，并在模型中纳人了温度、压力、间歇时间、瞬 时愈合、愈合和损伤历史以及损伤程度对材料愈合行 为的影响. 需要注意的是, 在该模型中, 他们认为, 当治 愈的时间尺度远大于损伤的时间尺度时, 愈合与损伤不 会同时发生. 该模型表现出时间和温度双重特性. 具体 的损伤演化方程和愈合演化方程如式(16)和(17)所示:

$$
\begin{aligned}
\dot{D}_{i}= & \Gamma_{0}^{\mathrm{d}}\left(\frac{\overline{Y_{i}}}{Y_{i}^{\text {th }}}\right)^{q}\left(1-D_{i}\right)^{n} \exp \left[-c_{1}\left(1-\frac{T}{T_{0}}\right)\right], \\
\dot{h}_{i}= & \Gamma_{0}^{\mathrm{h}}\left(1-h_{i}\right)^{m_{1}}\left(1-D_{i}\right)^{m_{2}}\left(1-s_{N}\right)^{m_{3}} \\
& \times \exp \left[-c_{2}\left(1-\frac{T}{T_{0}}\right)\right],
\end{aligned}
$$

其中, $\Gamma_{0}^{\mathrm{d}}$ 和 $\Gamma_{0}^{\mathrm{h}}$ 分别表示基准温度 $T_{0}$ 下损伤和愈合的流 动性参数, $c_{1}$ 和 $c_{2}$ 分别表示损伤和愈合温度耦合模型参 数, $Y_{i}^{\text {th }}$ 表示临界损伤值, $\bar{Y}_{i}$ 表示损伤驱动力. 值得注意 的是，与之前的演化方程不同，式(17)中引人了裂纹愈 合项 $\left(1-s_{N}\right)^{m_{3}}$, 表明施加一定荷载可以促进裂纹愈合. $m_{1}, m_{2}, m_{3}, q, n$ 分别表示材料参数.

Darabi等人 ${ }^{[74}$ 提出了考虑黏弹塑性效应的沥青混 凝土损伤愈合本构模型. 该模型基于Schapery ${ }^{[76]}$ 的非线 性黏弹性(viscoelastic, VE)、Perzyna ${ }^{[77]}$ 的黏塑性(viscoplastic, VP), 引人秥性损伤(viscodamage, VD)和微损 伤-愈合(healing, H)模型, 模拟了沥青混凝土在瘦劳过 程中的非线性力学响应. 首先, 基于小变形理论, 利用 加法规则将总应变张量 $\varepsilon$ 分解为黏弹性应变张量 $\boldsymbol{\varepsilon}^{\mathrm{ve}}$ 和 黏塑性应变张量 $\varepsilon^{\mathrm{vp}}$. 然后, 在Schapery黏弹性模型及Lai 和Bakker ${ }^{[78]}$ 研究基础上, 得到黏弹性应变偏张量 $\left(\varepsilon_{i j}^{\mathrm{ve}}\right)$ 和球张量 $\left(\varepsilon_{k k}^{\mathrm{ve}}\right)$ 的表达式. 黏塑性应变率张量 $\dot{\varepsilon}^{\mathrm{vp}}$ 是在 Perzyna $^{[77]}$ 提出的黏塑性应变模型和改良的DruckerPrager函数的基础上得到的.

对 Abu Al-Rub等人 ${ }^{[67]}$ 提出的黏性损伤模型进行修 正, 用幂函数替代原本有效应变中的指数形式, 得到黏 性损伤模型:

$\dot{\phi}=\Gamma^{\mathrm{vd}}\left(\frac{Y}{Y_{0}}\right)^{q}\left(\varepsilon_{\mathrm{eff}}\right)^{k}$,

其中, $\dot{\phi}$ 是损伤密度速率; $\Gamma^{\mathrm{vd}}$ 是黏性损伤流动性参数; $Y$ 
是基准损伤力; $\varepsilon_{\mathrm{eff}}$ 是总有效应变, 被定义为 $\varepsilon_{\mathrm{eff}}=\sqrt{\varepsilon_{i j} \varepsilon_{i j}} ; Y$ 是损伤力; $k$ 和 $q$ 是材料参数.

微损伤-愈合模型则是基于 $\mathrm{Abu} \quad \mathrm{Al}-\mathrm{Rub}^{[73]}$ 在2010 年的研究, 如式(19)所示:

$\dot{h}=\Gamma^{\mathrm{h}}(1-\phi)^{b_{1}}(1-h)^{b_{2}}$,

其中, $\Gamma^{\mathrm{h}}$ 是微损伤愈合流动参数; $b_{1}$ 和 $b_{2}$ 分别表示损伤 和微损伤愈合历史参数; $\phi$ 是损伤变量; $h$ 是愈合变量. 为了减少参数数量, 该模型可以直接简化为最简单的 演化函数:

$\dot{h}=\Gamma^{\mathrm{h}}$.

该模型通过大量的实验数据进行验证，包括恒定应变 率、循环位移控制和循环应力控制测试. 对比结果表 明，该模型能够预测不同加载条件下沥青混凝土的疲 劳损伤响应.

Oucif等人 ${ }^{[79]}$ 提出了一种超级愈合理论. 超级愈合 被定义为, 在材料已经恢复到原始力学性能后, 愈合机 制仍继续发生，从而起到加固材料的作用，如图4所示. 依据超级愈合概念, 式(1)被进一步扩展, 如式(21)所示: $\sigma=\bar{\sigma}\left[1+h_{\mathrm{s}}(n+1)-1\right] \varphi$,

其中, $h_{\mathrm{s}}(n+1)$ 被定义为超级愈合变量, 并可以分为单 一超级愈合机制和复合超级愈合机制. 在单一愈合机 制下， $n$ 通常取 1 , 因为只有单一的超级愈合变量被激 发. 这种机制可以应用到基于微胶囊材料自愈合的情 况中. 复合超级愈合机制下, $n$ 大于 1 , 它的取值取决于 超级愈合和愈合发生的点，这种机制可以发生在具有 多重裂缝的损伤材料的自愈合中.

Oucif等人 ${ }^{[79]}$ 基于弹性刚度变量恢复和强化，分析 了线性、广义非线性和超级愈合二次方程(quadratic super healing, QSH)的特性. 分别应用弹性应变等效假 设和弹性应变能等效假设, 比较了线性愈合效率和超 愈合效率变量，如表1所示，并将线性超愈合扩展到基 于弹性刚度强化的广义非线性和广义弹性刚度理论, 对两种理论的超愈合行为进行了比较. 结果表明, 弹性 能量等效假设高估了广义非线性和QSH理论的超愈合 弹性刚度强化. 同时, 证明了广义非线性和QSH理论都 适用于平面应力的情况. 此外，与QSH理论相比，广义 非线性超级愈合(generalized nonlinear super healing,

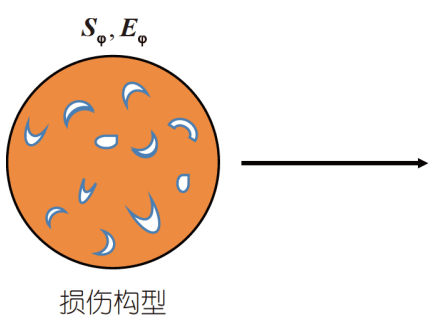

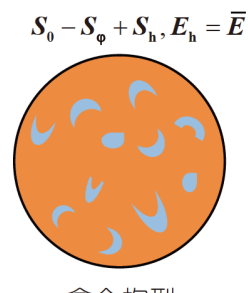

愈合构型

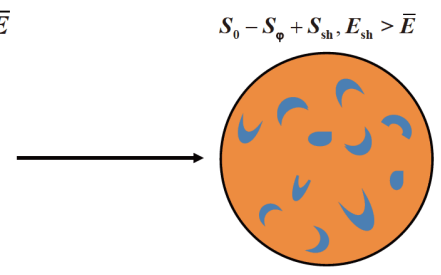

超级愈合构型

图 4 (网络版彩色)损伤、愈合以及超级愈合下材料的状态

Figure 4 (Color online) States of the materials under damage, healing, and super healing

表 1 弹性应变等效假设和弹性应变能等效假设下损伤、愈合及超级愈合的弹性模量 ${ }^{a)}$

Table 1 Elastic modulus in damage, healing or super healing configuration under the assumption of elastic strain equivalence or elastic strain energy equivalence

\begin{tabular}{|c|c|c|c|c|}
\hline & \multicolumn{2}{|c|}{ 弹性应变等效假设 } & \multicolumn{2}{|c|}{ 弹性能等效假设 } \\
\hline & 弹性模量 & 变量 & 弹性模量 & 变量 \\
\hline 损伤 & $E_{\varphi}=\bar{E}(1-\varphi)$ & $\varphi=\frac{\bar{E}-E_{\mathrm{\varphi}}}{\bar{E}}$ & $E_{\varphi}=\bar{E}(1-\varphi)^{2}$ & $\varphi=1-\sqrt{\frac{E_{\varphi}}{\bar{E}}}$ \\
\hline 愈合 & $E_{\mathrm{h}}=\bar{E}[1-\varphi(1-h)]$ & $h=1-\frac{\bar{E}-E_{\mathrm{h}}}{\varphi \bar{E}}$ & $E_{\mathrm{h}}=\bar{E}[1-\varphi(1-h)]^{2}$ & $h=1-\frac{\sqrt{\bar{E}}-\sqrt{E_{\mathrm{h}}}}{\varphi \sqrt{\bar{E}}}$ \\
\hline 超级愈合 & $E_{\mathrm{sh}}=\bar{E}[1+(R-1) \varphi]$ & $R=\frac{E_{\mathrm{sh}}-\bar{E}}{\varphi \bar{E}}+1$ & $E_{\mathrm{sh}}=\bar{E}[1+(R-1) \varphi]^{2}$ & $R=\frac{\sqrt{E_{\mathrm{h}}}-\sqrt{\bar{E}}}{\varphi \sqrt{\bar{E}}}+1$ \\
\hline
\end{tabular}

a) $E_{\rho}, E_{\mathrm{h}}, E_{\mathrm{sh}}$ 和 $\bar{E}$ 分别是损伤、愈合、超级愈合以及有效构型中的弹性模量; $R$ 是超级愈合参数; $\varphi$ 和 $h$ 分别是损伤和愈合变量 
GNSH)理论在两种等效假设中都给出了超愈合弹性刚 度的高强化表现.

\section{4 考虑自愈合效应的数值模拟方法}

\section{1 分子动力学方法}

分子动力学(molecular dynamics, MD)可以帮助人 们从微观角度认识物质的基本特征，是一种从分子水 平对复杂体系进行研究的有效手段. 目前, 在土木工程 领域, 采用分子动力学方法进行材料自愈合行为的研 究主要以沥青为主, 在无机水泥基材料方面尚未推广, 这也为日后的研究方向提供了一个新的思路. 下文在 分子动力学方法的总结分析中, 仅针对励青材料自愈 合机制的应用.

Bhasin $^{[80]}$ 首次在沥青自愈合研究中采用分子动力 学方法, 以Jennings等人 ${ }^{[81]}$ 提出的沥青分子模型为基础, 在两个沥青分子模型之间设置 $50 \AA$ 间隙, 以代表荷载作 用下沥青材料产生的微裂纹. 对整个模型进行动力学 模拟后，从轨迹文件中提取两团沥青分子质心的均方 位移, 计算其随时间的运动和速度, 从而计算分子的扩 散系数. 模拟结果发现, 在 $100 \mathrm{ps}$ 模拟时间和 $873 \mathrm{~K}$ 模拟 温度下, 裂纹很快愈合, 证明可以通过补充能量(升温) 的方式促进裂纹愈合. 同时, 亚甲基和甲基中碳原子数 的比值 $\mathrm{CH}_{2} / \mathrm{CH}_{3}$ 越大, 即沥青分子链的长度越长, 扩散 系数越大，自愈合速率也越大，表明对沥青进行分子模 拟时, 其分子结构会影响愈合速率的大小; 亚甲基和甲 基中氢原子数与碳原子数的比值MMHC越大，即沥青 分子结构中的侧链分支越多, 裂纹间的扩散系数越小.

Shen等人 ${ }^{[82]}$ 利用开源软件LAMMPS建立了不同温 度和不同裂㖓宽度的沥青分子MD模型. 模拟时采用 Reax力场, 该力场可描述化学键的连续断裂和重整, 定 义动力学参数, 并计算非键相互作用, 如范德华力和库 仑相互作用力, 能够更好地研究沥青分子的自愈合机 理. 结果表明, 沥青分子的扩散机制可引发自愈合, 温 度越高, 分子扩散率越高, 愈合速率越高.

$\mathrm{Xu}$ 和Wang ${ }^{[83]}$ 利用分子动力学建立了含 $10 \AA$ 裂纹 的两层沥青模型, 利用不同含量的饱和分、芳香分、 胶质和沥青质, 构建了老化前后沥青分子模型. 通过各 种热力学性质对分子模型进行验证，包括密度、表面 能、黏度和内聚能. 采用径向分布函数和均方位移对 基质沥青和老化沥青分子结构进行了比较. 模拟并计 算了不同温度下老化沥青和未老化沥青的分子扩散能
力和活化能. 结果表明, 沥青扩散系数随温度升高而增 大, 与原沥青相比, 老化沥青具有较低的分子扩散速率 和较高的自愈活化能屏障.

Sun等人 ${ }^{[84]}$ 利用Materials Studio分子动力学模拟软 件构建了基质沥青、SBS改性沥青的分子模型，如图5 所示, 将沥青分子模型的密度、玻璃态转化温度与实 测数据进行比较, 验证了沥青分子模型的准确性和可 行性. 通过Arrhenius方程, 计算沥青分子动力学模型愈 合过程的活化能和指前因子. 活化能反映沥青分子扩 散对模拟温度的敏感性和反应的最小能量，指前因子 意味着参与反应的沥青分子数量. SBS改性沥青的活 化能大于基质沥青，这是因为改性剂阻碍了沥青分子 之间的连接，沥青分子需克服较大的能量才能实现裂 纹间的自由扩散、排列组合，而且温度对沥青分子的 运动轨迹也会产生较大的影响. SBS改性沥青的指前 因子显著大于基质沥青，说明添加改性剂分子促使沥 青分子参与愈合行为.

\section{2 有限元法}

\subsection{1 自愈合触发机制}

在采用胶囊/纤维的自愈系统 ${ }^{[85 ~ 88]}$ 中, 需要首先确 保胶囊或纤维能够在裂纹产生时及时发挥作用. 以自 愈合胶囊为例, 裂纹尖端能否适时触碰并打开胶囊, 促 使其释放出愈合剂是成功触发自愈合的重要前提. 围 绕这一点, 研究者们从损伤与断裂力学角度出发, 借助 数值模拟方法对裂纹的起裂、扩展以及胶囊的破裂过 程进行了分析. Zemskov等人 ${ }^{[89]}$ 提出了一种二维解析模 型，通过分析裂纹长度、胶囊尺寸和平均胶囊间距等 参数, 计算裂纹触碰到胶囊的概率，间接估算出自愈合 效率. Gilabert等人 ${ }^{[90]}$ 和Xue等人 ${ }^{[91]}$ 基于扩展有限元法, 分别分析了管状和球状自愈合胶囊在混凝土材料中的 界面性能和断裂机制. 研究发现, 胶囊-基体的界面过渡 区强度对胶囊的破裂有显著的影响. 当裂纹扩展至胶 囊时, 弱界面可能会导致脱黏现象的发生, 使得胶囊无 法发生破裂. 其研究结果对自愈合胶囊的设计(如几何 分布、材料特性及界面性能等)具有指导意义. Maulu$\operatorname{din}$ 和Oucif ${ }^{[92]}$ 借助有限元法着重分析了胶囊界面的影 响, 讨论了胶囊的裂纹形核、扩展和断裂方式, 发现胶 囊在自愈合混凝土中的断裂概率与核壳厚度比密切相 关. 在有限元模型中, 胶囊-基体的界面区通常由内聚力 单元(cohesive element)或内聚力表面(cohesive surface) 来实现, 其损伤模型多采用线性牵引-分离准则. 

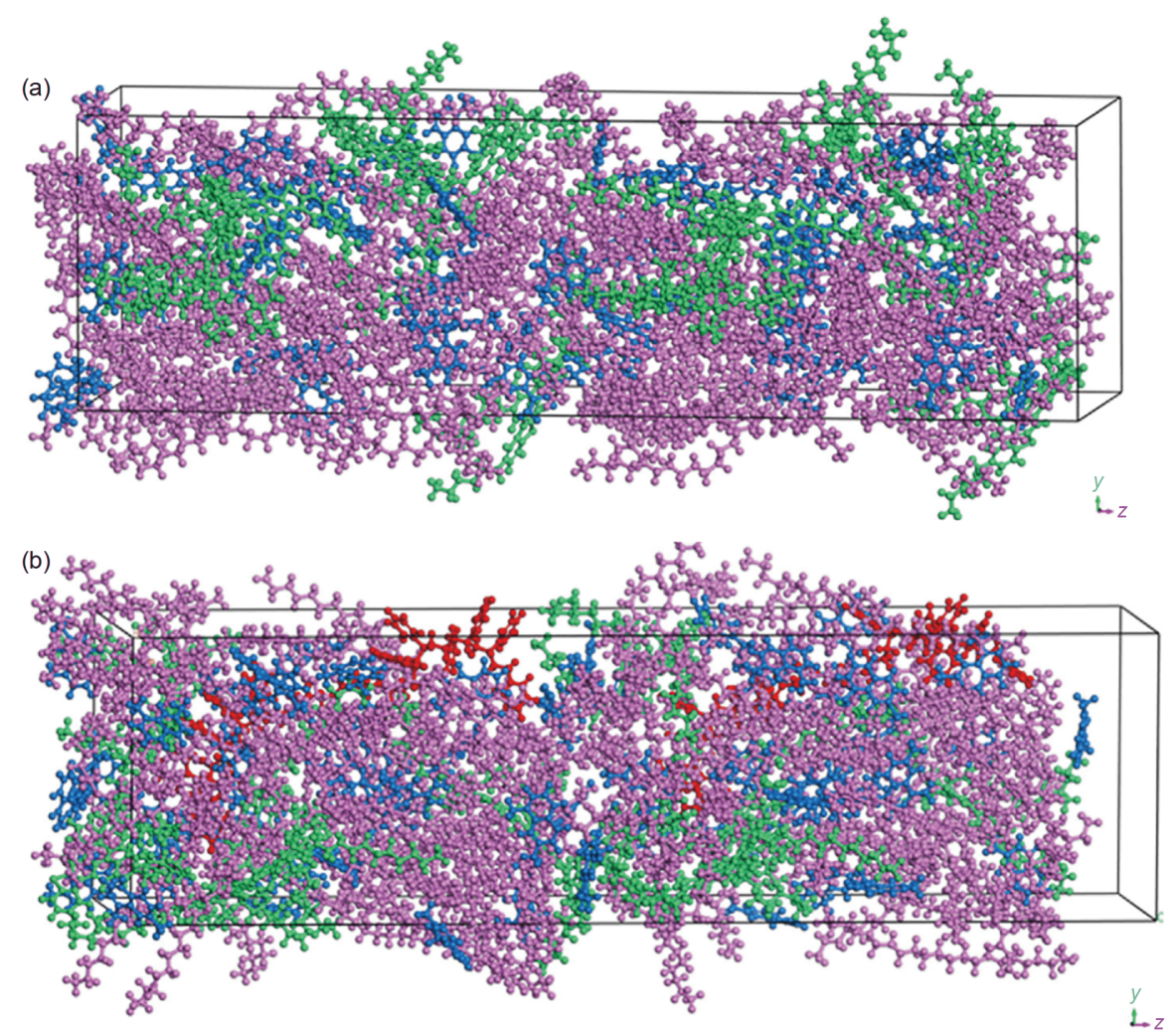

图 5 (网络版彩色)动力学模拟后的层间结构 ${ }^{[84]}$. (a) 基质沥青; (b) SBS改性沥青

Figure 5 (Color online) Layer structure after dynamics simulation ${ }^{[84]}$. (a) Neat asphalt; (b) SBS modified asphalt

\subsection{2 自愈合材料的恢复机制}

在确保自愈合能被成功激活的前提下，明确材料 力学性能的恢复机制是自愈合研究的重中之重.

(1) 基于CDHM模型的有限元数值分析. CDHM模 型通过直接在材料本构模型中引人损伤变量和愈合变 量来实现材料的损伤愈合过程. Abu Al-Rub等人 ${ }^{[67]}$ 通 过在黏弹塑性本构模型中引人损伤变量和愈合变量, 构建了CDHM模型，其中损伤及愈合演化方程考虑了 加载速率和时间的影响. 在进一步的有限元分析中, 基 于用户子程序UMAT来完成上述复杂本构模型的数值 实现，分别采用递归-迭代和径向返回算法进行模型的 黏弹性和黏塑性部分的计算，而黏性损伤和愈合部分 则采用等效构型的概念. 针对沥青混合料设计了重复 蠕变-恢复加载试验, 并进行了相应的有限元虚拟实验. 仿真和试验对比结果表明，在材料模型中同时考虑损 伤和愈合效应, 才能更好地表征实际材料的力学行为. 该模型相比于传统损伤模型, 能够更加精准地进行沥 青混合料性能(如疲劳寿命)的预测.

Darabi等人 ${ }^{[63]}$ 对应变等效假设、弹性应变能等效
假设以及功率等效假设进行了对比分析，介绍了基于 不同转换假设的数值实现过程. 值得注意的是, 如果采 用应变等效假设, 则名义应变张量及其增量将与损伤 愈合构型中的相同; 然而, 如果采用弹性应变能等效或 功率等效假设, 则名义应变张量及其增量将与其对应 的值不同. 因此, 当采用弹性应变能等效假设或功率等 效假设时, 需要采用迭代方法获得 $t+\Delta t$ 时刻愈合构型中 的总应变和应变增量张量, 具体实现过程如图6所示. 在单轴恒定应力速率和单轴恒定应变速率加载下，得 到了基于不同假设所对应的材料割线刚度模量的预测 结果. 结果表明, 当使用应变或弹性应变能假设时, 割 线刚度模量与路径无关; 而当使用功率等效假设时, 割 线刚度模量取决于加载历史. 因此, 从物理意义上来说, 功率等效假设更为合理.

（2）考虑自愈合效应的多尺度分析. CDHM被广泛 应用于裂纹的愈合分析, 然而, 该方法缺乏从物理微观 层面对材料损伤-断裂-愈合机理的解释. 衔接微观机理 和宏观响应的多尺度分析方法能够更加系统地诠释与 表征断裂-愈合模型. Shojaei等人 ${ }^{[93]}$ 以内嵌固体愈合剂 


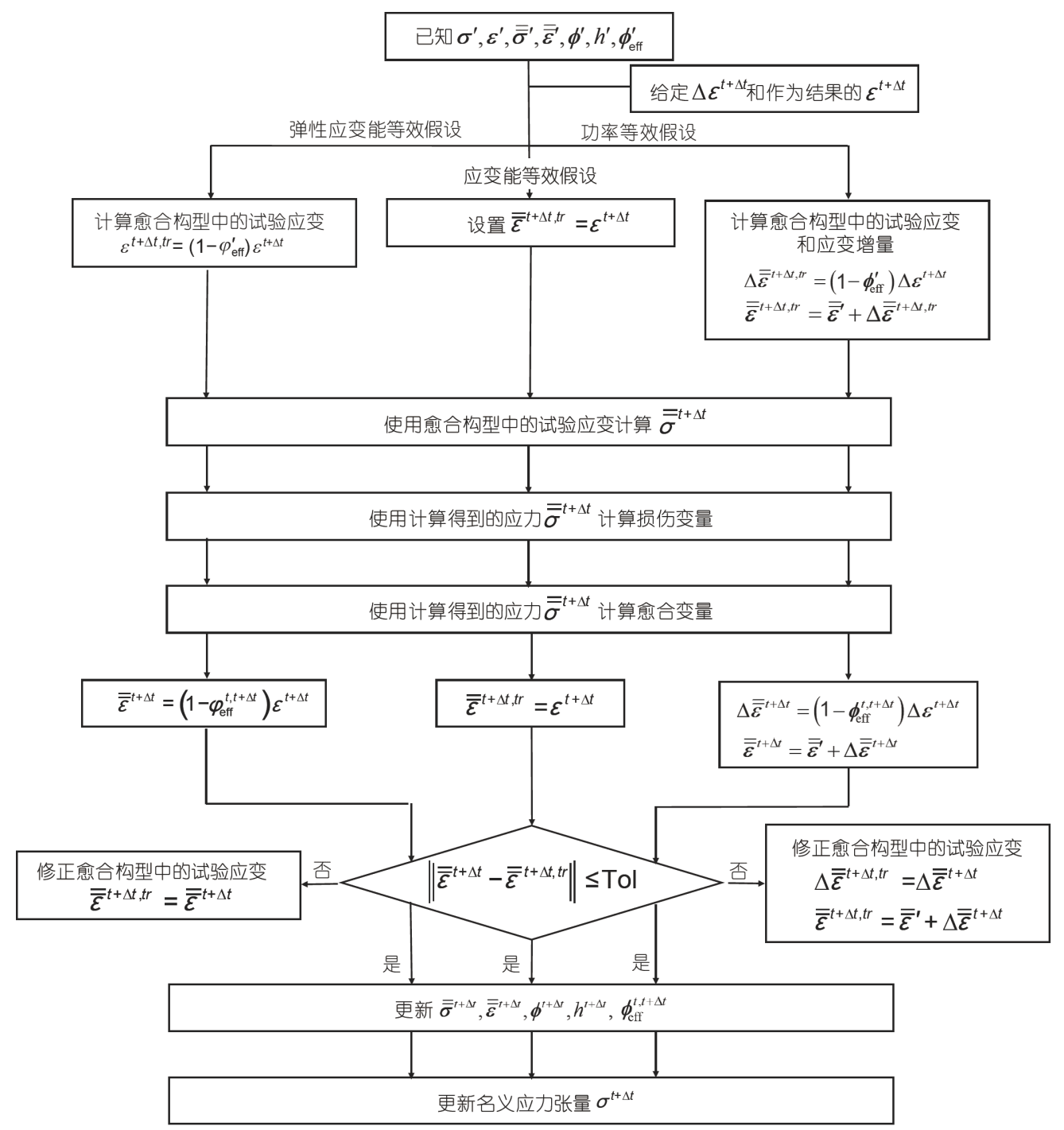

图 6 使用不同转换假设的弹性损伤-愈合模型的一般有限元实现过程

Figure 6 A general finite element implementation process using different transformation assumptions for elastic damage-healing models

的自愈合复合材料为研究对象, 提出了一种多尺度愈 合本构模型. 在损伤发生后, 作为基质的形状记忆聚合 物(shape memory polymer, SMP)和作为固体愈合剂的 热塑性颗粒(thermoplastic particle, TP)同时发挥作用以 促使材料发生愈合. 当裂纹产生后, 首先由具备形状记 忆特性的SMP基质通过形状恢复致使裂纹面发生闭合， 再由TP愈合剂充分浸润裂纹面而实现最终的愈合，这 一过程被称为裂纹闭合后愈合(close-then-heal, CTH) 过程. 多尺度愈合框架将微观力学和CDHM模型相结 合, 可以将分子水平的愈合机制(浸润和扩散)与宏观力 学响应(材料刚度和强度恢复)相关联.

Shojaei等人 ${ }^{[93]}$ 还构建了SMP-TP复合材料的三维
有限元模型，其材料模型部分基于上述理论通过编写 UMAT实现．在愈合过程中，应力、应变、温度和时间 的状态量被传递到UMAT中，以计算浸润和扩散参数 并相应地更新愈合参数，更新的刚度矩阵被用于计算 新的应力场和应变场增量. 愈合参数的结果云图表明, 愈合效率同时受边缘处的热损耗和接触断裂面上的不 均匀压力梯度影响.

（3）考虑愈合效应的内聚力模型. Ponnusami等 人 $^{[94]}$ 提出了一种用于表征复合材料开裂愈合过程的本 构模型，该模型可借助内聚力单元或扩展有限元法来 实现. 如图7所示，在裂纹尖端处内聚区的材料被区分 为未损伤开裂部分 $($ 标记为(0))和损伤开裂部分, 一旦愈 


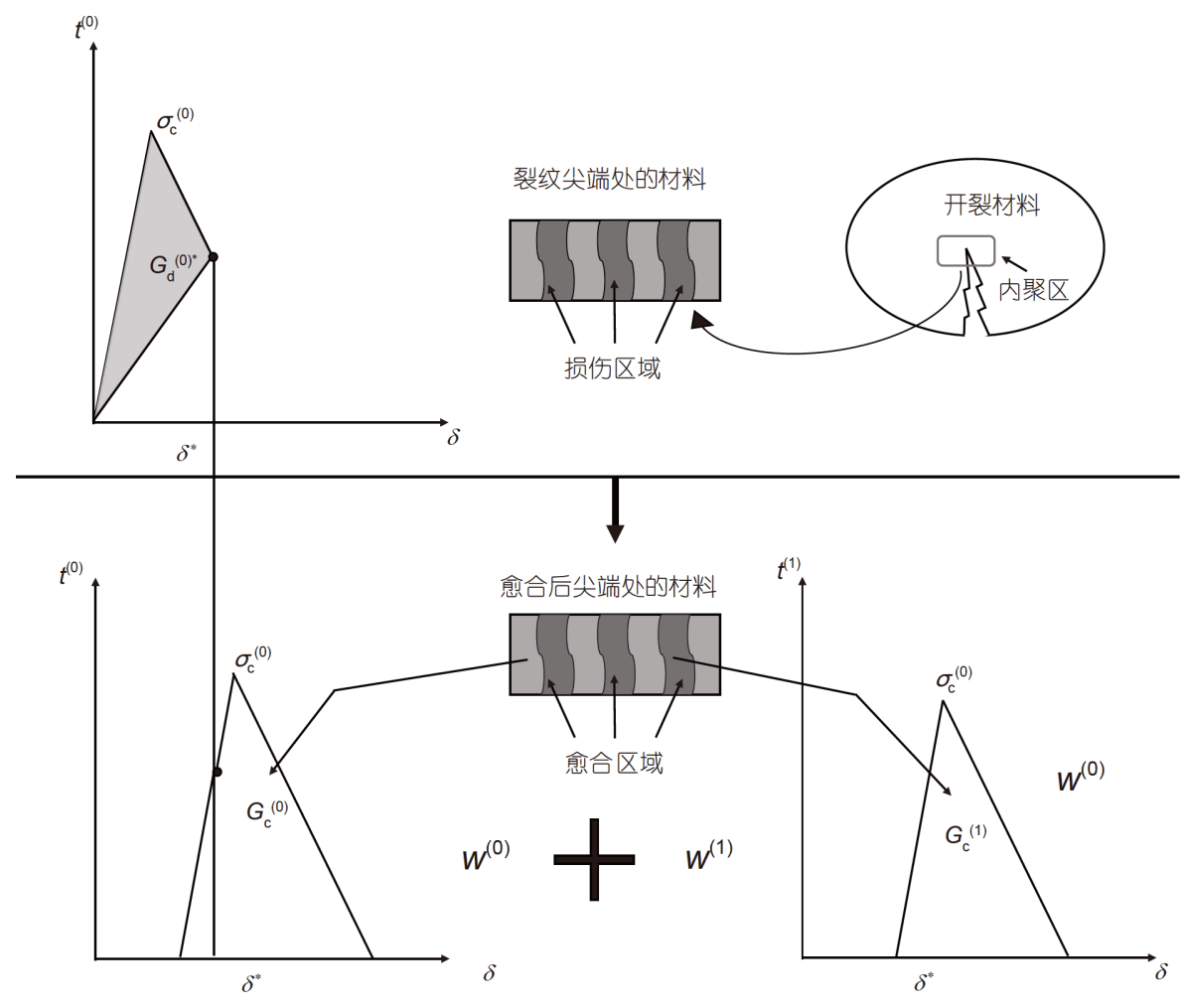

图 7 未损伤开裂部分材料与愈合部分材料的牵引-分离准则

Figure 7 Traction-separation law for undamaged and cracked parts and healed parts

合机制被激发, 损伤开裂部分发生愈合, 材料性能再次 发生改变(标记为(1)). 在后续受力过程中, 未损伤开裂 部分材料仍遵循初始材料的牵引-分离准则，而愈合部 分由于损伤的弱化作用而遵循新的牵引-分离准则. 复 合材料在愈合后的有效断裂能 $\widetilde{G}_{\mathrm{c}}$ 被定义为未损伤部分 材料断裂能 $\left(G_{\mathrm{c}}^{(0)}\right)$ 和愈合部分材料断裂能 $\left(G_{\mathrm{c}}^{(1)}\right)$ 按照表 面体积分数 $(w)$ 计算得到的加权和, 即 $\widetilde{G}_{\mathrm{c}}=w^{\left({ }^{(0)}\right.} G_{\mathrm{c}}{ }^{(0)}+$ $w^{(1)} G_{\mathrm{c}}^{(1)}$.

该研究中, 内聚区的牵引-分离准则具体采用的是

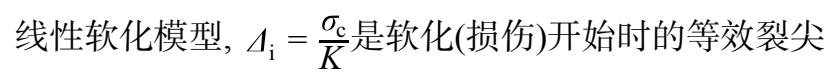
位移, $\Delta_{\mathrm{f}}=\frac{2 G_{\mathrm{c}}}{\sigma_{\mathrm{c}}}$ 是软化(损伤)开始时的最大等效裂尖位 移. 在使用Newton-Raphson迭代法进行有限元求解的 过程中, 内聚力单元的切线矩阵表示为牵引力对裂纹 张开位移导数的加权和, 即

$\frac{\partial \tilde{t}_{i}^{[m]}}{\partial \delta_{j}}=\sum_{p=0}^{m} w^{[m](p)} \frac{\partial t_{i}^{(p)}}{\partial \delta_{j}}, i=n, s, j=n, s$,
其中, $\delta$ 是裂纹张开位移, $t$ 是牵引力, $p$ 是自愈合发生的 次数 $(p \in[0, m])$,下标 $n$ 和 $s$ 分别表示法向和切向的分量.

采用有限元法，在两个二维平板间引人零厚度的 内聚力单元并进行单轴拉伸加载, 以此验证上述内聚 力模型. 加载历史涉及部分损伤、愈合、部分损伤和 卸载四个阶段, 仿真结果证实了上述内聚力模型的合 理性和有限元算法的正确性.

Alsheghri和 $\mathrm{Abu} \mathrm{Al}-\mathrm{Rub}^{[95]}$ 在内聚力模型中引入 CHDM理论, 提出内聚区损伤愈合力学模型(cohesive zone damage-healing model, CZHDM). 他们将材料分 为两部分, 即采用CZHDM模型的损伤-愈合部分和普 通材料本构(即弹性、黏弹性或黏弹塑性)部分. 当确定 损伤和愈合演化方程后，在原材料的双线性牵引-分离 准则中引人损伤和愈合变量，从而构建CZHDM模型. 图8给出了该模型有限元数值算法的流程图, 相应的材 料模型通过编写UMAT来实现. 通过将紧凑拉伸虚拟 试验结果与实际试验结果进行对比，表明该模型能合 理地描述材料的力学特性. 除此之外, 他们研究了间歇 时间对材料内聚力模型的影响, 结果显示, 间歇时间越 


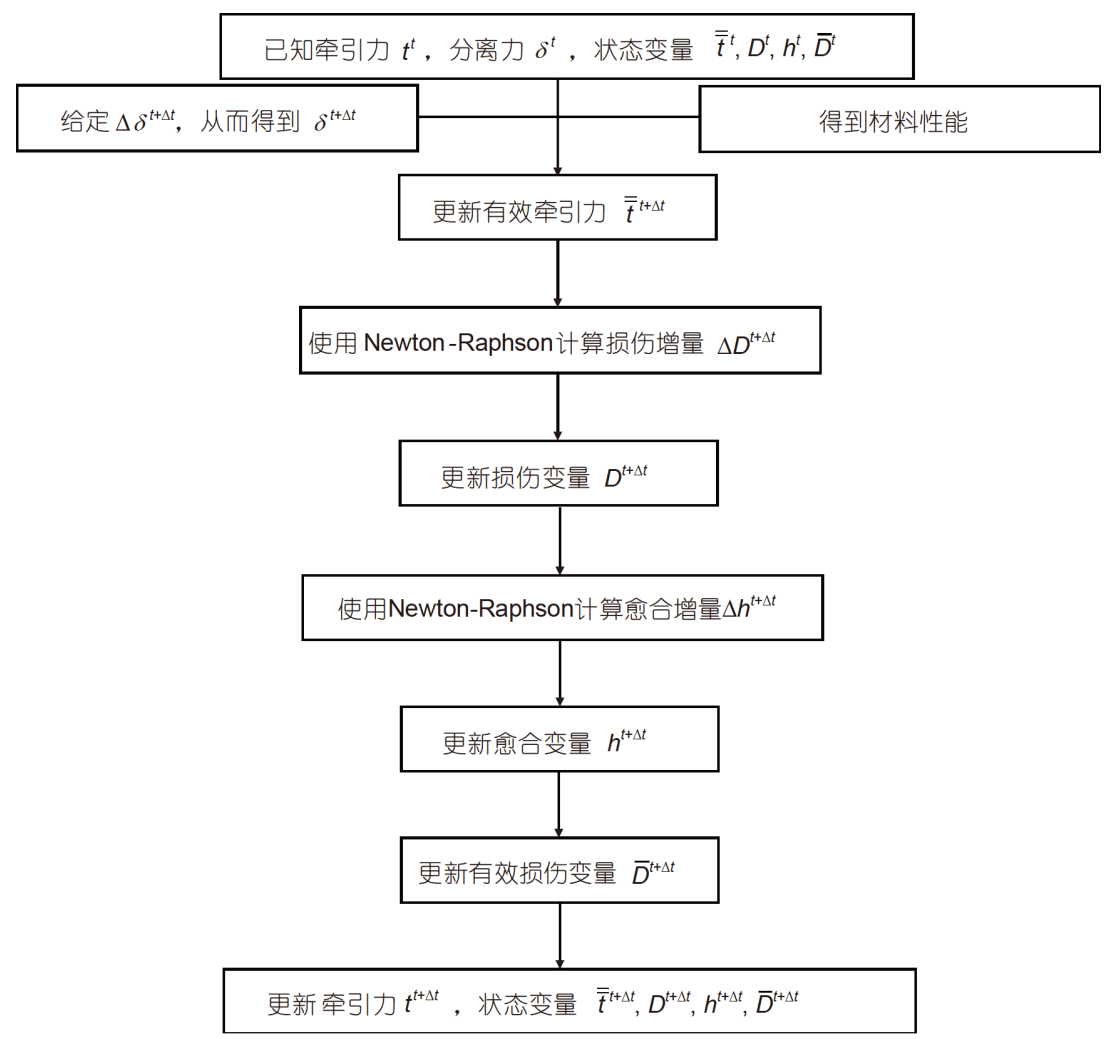

图 8 CZDHM模型数值算法流程图

Figure 8 A flow chart of CZDHM model numerical algorithm

长, 内聚区材料的整体性能恢复越多, 自愈合效果越好.

\section{5 总结与展望}

自愈合效应对裂纹起裂点、起裂时间、裂纹扩展 路径、裂纹扩展速率等均有极为重要的影响，进而左 右着材料的实际使用寿命. 为此, 构建考虑材料自愈合 特性的损伤本构模型及相应的快速数值算法对理解自 愈合机理、精准预估裂纹的扩展轨迹、精确预测材料 的使用寿命意义重大. 目前, 自愈合工程材料在应用过 程中还存在着若干力学问题有待解决: (1) 从力学角度 来讲, 损伤意味着力学性能的衰减, 而开裂则表现为裂 纹处位移的不连续性. 显然, 在构建考虑自愈合效应的 本构模型时，应加以区别对待. (2) 材料的实际㽻劳寿 命关系到损伤演化、裂纹扩展与自愈合效应三者的共 同作用. 因此, 所建立的瘦劳模型应能够同时将这三者 考虑在内. (3) 考虑到很多自愈合工程材料，如沥青混 合料是典型的黏弹塑性材料，如何将黏弹性效应及自 愈合效应对宏观性能恢复的影响进行解耦，需要进一
步研究. (4) 上述的疲劳破坏过程较为复杂, 尤其要在 一个力学模型中体现出损伤-自愈和开裂-自愈是非常 困难的, 有效的途径是建立同时兼容损伤-自愈和开裂自愈的数值模型. (5) 材料宏观表现出来的损伤、断裂 和愈合并非一蹴而就，而是一个由下而上、层层递进 的过程，涉及纳观、微观、细观、宏观等多个尺度之 间的联动. 因此, 要深刻地理解材料的愈合与破坏, 就 不能仅仅停留在给出表象的宏观尺度上，而应充分地 开展多尺度分析.

自愈合材料在提升工程结构耐久性及可靠性上冊 庸置疑将发挥重要作用. 但是, 要真正实现自愈合材料 在土木工程中的大范围使用，其关键还在于大幅度提 升自愈合效率. 为此, 还需从力学角度出发, 从本质上 理解自愈合行为，明确各内外因素对裂纹扩展-愈合的 正负耦合效应，为自愈复合材料的完善提供强有力的 理论基础, 并开发快速数值算法, 精准预测自愈合效应 影响下的服役寿命，为自愈复合材料的设计、使用、 潜力挖掘提供高精、高效的分析手段. 


\section{参考文献}

1 de Rooij M, Van Tittelboom K, De Belie N, et al. Self-healing Phenomena in Cement-based Materials. Dordrecht: Springer Netherlands, 2013

2 Ramm W, Biscoping M. Autogenous healing and reinforcement corrosion of water-penetrated separation cracks in reinforced concrete. Nucl Eng Des, 1998, 179: 191-200

3 Zhang W, Zheng Q, Ashour A, et al. Self-healing cement concrete composites for resilient infrastructures: A review. Compos Part B-Eng, 2020, 189: 107892

4 Little D N, Bhasin A. Self Healing Materials. Berlin: Springer-Verlag, 2007

5 Daniel J S, Kim Y R. Laboratory evaluation of fatigue damage and healing of asphalt mixtures. J Mater Civil Eng, 2001, 13: 434-440

6 Tan Y, Shan L, Richard Kim Y, et al. Healing characteristics of asphalt binder. Constr Build Mater, 2012, 27: 570-577

7 Bonnaure F P, Huibers A H, Boonders A. A laboratory investigation of the influence of rest periods on the fatigue characteristics of bituminous mixes. In: Journal of the Association of Asphalt Paving Technologists. Washington: The National Academies of Sciences, Engineering, and Medicine, 1982. 104-128

8 Garcia S J. Effect of polymer architecture on the intrinsic self-healing character of polymers. Eur Polym J, 2014, 53: 118-125

9 Homma D, Mihashi H, Nishiwaki T. Self-healing capability of fibre reinforced cementitious composites. J Adv Concr Technol, 2009, 7: 217-228

10 Feng J, Su Y, Qian C. Coupled effect of PP fiber, PVA fiber and bacteria on self-healing efficiency of early-age cracks in concrete. Constr Build Mater, 2019, 228: 116810

11 Sun D S, Chen Y Y, Wang A G, et al. Research progress of self-healing and self-repairing concrete (in Chinese). Mater Rep, 2014, 28: 132-136 [孙 道胜, 陈远远, 王爱国, 等. 自愈合和自修复混凝土的研究进展. 材料导报, 2014, 28: 132-136]

12 Feng J J, Zhang P, Chen W, et al. Self-healing behavior of early concrete cracks incorporating magnesium oxide expansive agent (in Chinese). J Build Mater, 2018, (4): 656-662 [冯竟竟, 张鹏, 陈伟, 等. 氧化镁膨胀剂对混凝土早期裂缝自愈合性能的影响. 建筑材料学报, 2018, (4): 656662]

13 Qureshi T S, Al-Tabbaa A. The effect of magnesia on the self-healing performance of Portland cement with increased curing time. In: 1st International Conference on Ageing of Materials and Structures. Delft, 2014. 635-642

14 Sherir M A A, Hossain K M A, Lachemi M. The influence of MgO-type expansive agent incorporated in self-healing system of engineered cementitious Composites. Constr Build Mater, 2017, 149: 164-185

15 Snoeck D, Van Tittelboom K, De Belie N, et al. The use of superabsorbent polymers as a crack sealing and crack healing mechanism in cementitious materials. In: Alexander M G, Beushausen H D, Dehn F, et al, eds. 3rd International Conference on Concrete Repair. Cape Town, 2012. $152-157$

16 Snoeck D, Van Tittelboom K, Steuperaert S, et al. Self-healing cementitious materials by the combination of microfibres and superabsorbent polymers. J Intell Mater Syst Struct, 2014, 25: 13-24

17 Snoeck D. Self-healing and microstructure of cementitious materials with microfibres and superabsorbent polymers. Struct Eng, 2015, doi: 10.13140/RG.2.1.4049.6086

18 Snoeck D, Steuperaert S, Van Tittelboom K, et al. Visualization of water penetration in cementitious materials with superabsorbent polymers by means of neutron radiography. Cement Concrete Res, 2012, 42: 1113-1121

19 Snoeck D, Van den Heede P, Van Mullem T, et al. Water penetration through cracks in self-healing cementitious materials with superabsorbent polymers studied by neutron radiography. Cement Concrete Res, 2018, 113: 86-98

20 Snoeck D. Superabsorbent polymers to seal and heal cracks in cementitious materials. RILEM Tech Lett, 2018, 3: 32-38

21 Snoeck D, De Belie N. Repeated autogenous healing in strain-hardening cementitious composites by using superabsorbent polymers. J Mater Civil Eng, 2016, 28: 04015086

22 Shen S, Carpenter S H. Dissipated Energy Concepts for HMA Performance: Fatigue and Healing. Technical Report, Urbana: University of Illinois. 2006

23 Huang W, Lv Q, Xiao F. Investigation of using binder bond strength test to evaluate adhesion and self-healing properties of modified asphalt binders. Constr Build Mater, 2016, 113: 49-56

24 Santagata E, Baglieri O, Tsantilis L, et al. Fatigue and healing properties of nano-reinforced bituminous binders. Int J Fatigue, 2015, 80: 30-39

25 Carpenter S H, Shen S. Dissipated energy approach to study hot-mix asphalt healing in fatigue. Transp Res Record, 2006, 1970: 178-185

26 Shen S, Chiu H M, Huang H. Characterization of fatigue and healing in asphalt binders. J Mater Civil Eng, 2010, 22: 846-852

27 Van Den Bergh W. The effect of ageing on the fatigue and healing properties of bituminous mortars. Doctor Dissertation. Delft: Delft University of Technology, 2011

28 Kim B, Roque R. Evaluation of healing property of asphalt mixtures. Transp Res Record, 2006, 1970: 84-91 
29 Grant T P. Determination of asphalt mixture healing rate using the superpave indirect tensile test. Master Dissertation. Florida: University of Florida, 2001

30 Huang H L, Ye G, Shui Z H. Feasibility of self-healing in cementitious materials—By using capsules or a vascular system? Constr Build Mater, 2014, 63: 108-118

31 Dry C. Procedures developed for self-repair of polymer matrix composite materials. Compos Struct, 1996, 35: 263-269

32 Van Tittelboom K, De Belie N, Van Loo D, et al. Self-healing efficiency of cementitious materials containing tubular capsules filled with healing agent. Cement Concrete Compos, 2011, 33: 497-505

33 García A, Schlangen E, van de Ven M, et al. Preparation of capsules containing rejuvenators for their use in asphalt concrete. J Hazard Mater, 2010, 184: 603-611

34 Sun D, Pang Q, Zhu X, et al. Enhanced self-healing process of sustainable asphalt materials containing microcapsules. ACS Sustain Chem Eng, 2017, 5: 9881-9893

35 Gao L X, Sun G W. Development of microbial technique in self-healing of concrete cracks (in Chinese). J Chin Ceram Soc, 2013, 41: 627-636 [高 礼雄, 孙国文. 微生物技术在混凝土裂缝自修复中应用的研究进展. 硅酸盐学报, 2013, 41: 627-636]

36 Qian C X, Li R Y, Pan Q F. Microbial self-healing effects of concrete cracks (in Chinese). J Southeast Univ (Nat Sci Ed), 2013, 43: 360-364 [钱春 香, 李瑞阳, 潘庆峰, 等. 混凝土裂缝的微生物自修复效果. 东南大学学报(自然科学版), 2013, 43: 360-364]

37 Jeremy L D, Ramakrishnan V, Sookie S B. Microbiologically induced sealant for concrete crack remediation. In: Proceedings of the $16 \mathrm{~h} \mathrm{ASCE}$ Engineering Mechanics Conference. Seattle: University of Washington Press, 2003. 93-111

38 Wang J, Van Tittelboom K, De Belie N, et al. Use of silica gel or polyurethane immobilized bacteria for self-healing concrete. Constr Build Mater, 2012, 26: 532-540

39 Wang J Y, Snoeck D, Van Vlierberghe S, et al. Application of hydrogel encapsulated carbonate precipitating bacteria for approaching a realistic self-healing in concrete. Constr Build Mater, 2014, 68: 110-119

40 Erşan Y Ç, Da Silva F B, Boon N, et al. Screening of bacteria and concrete compatible protection materials. Constr Build Mater, 2015, 88: 196-203

41 Singh H, Gupta R. Cellulose fiber as bacteria-carrier in mortar: Self-healing quantification using UPV. J Build Eng, 2020, 28 : 101090

42 Ryu J S, Otsuki N. Crack closure of reinforced concrete by electrodeposition technique. Cement Concrete Res, 2002, 32: 159-164

43 Otsuki N, Ryu J S. Use of electrodeposition for repair of concrete with shrinkage cracks. J Mater Civil Eng, 2001, 13: 136-142

44 Ryou J S, Monteiro P. Electrodeposition as a rehabilitation method for concrete materials. Can J Civil Eng, 2004, 31: 776-781

45 Ryou J S, Otsuki N. Experimental study on repair of concrete structural members by electrochemical method. Scripta Mater, 2005, 52: 1123-1127

46 Tao B Q, Liang D K, Xiong K, et al. Study on self-diagnosis and self-repairing function of shape memory alloy enhanced composite structure (in Chinese). Acta Aeronaut Et Astronaut, 1998, (2): 3-5 [陶宝祺, 粱大开, 熊克, 等. 形状记忆合金增强智能复合材料结构的自诊断、自修复功能 的研究. 航空学报,1998, (2): 3-5]

47 Zhang Y, Cui D, Guan P. Progress research on the shape memory alloy smart concrete structure (in Chinese). J Dalian Univ, 2012, 33: 23-28 [张岩, 崔迪, 关萍. 形状记忆合金智能混凝土结构研究进展. 大连大学学报, 2012, 33: 23-28]

48 Kuang Y, Ou J. Self-repairing performance of concrete beams strengthened using superelastic SMA wires in combination with adhesives released from hollow fibers. Smart Mater Struct, 2008, 17: 25020-25027

49 Sun L, Liang D, Gao Q, et al. Analysis on factors affecting the self-repair capability of SMA wire concrete beam. Math Probl Eng, 2013, 2013: $469-481$

50 Zhou T S, Pei J Z, Li R, et al. The preparation and properties of road self-healing materials by light excitation (in Chinese). Synth Mater Aging App, 2015, 44: 45-50 [周天澍, 裴建中, 李芯, 等. 光激发路用自修复材料的制备与表征. 合成材料老化与应用, 2015, 44: 45-50]

51 Chen S L, Zhang Y Y, Huang W H, et al. Self-repairing technological research of concrete microcracks: Recent developments, future directions (in Chinese). Guangzhou Archit, 2019, (5): 3-8 [陈胜利, 张译允, 黄文辉, 等. 混凝土微裂缝自修复技术研究进展及展望. 广州建筑, 2019, (5): 38]

52 Bazin P, Saunier J. Deformability, fatigue, and healing properties of asphalt mixes. In: Arbor A, ed. Proceedings of the Second International Conference on the Structural Design of Asphalt Pavements. Michigan, 1967. 53-69

53 Liu Q, Wu S, Schlangen E. Induction heating of asphalt mastic for crack control. Constr Build Mater, 2013, 41: 345-351

54 Liu X, Liu W, Wu S, et al. Effect of carbon fillers on electrical and road properties of conductive asphalt materials. Constr Build Mater, 2014, 68: 301-306

55 Dai Q, Wang Z, Mohd Hasan M R. Investigation of induction healing effects on electrically conductive asphalt mastic and asphalt concrete beams through fracture-healing tests. Constr Build Mater, 2013, 49: 729-737

56 Gómez-Meijide B, Ajam H, Lastra-González P, et al. Effect of air voids content on asphalt self-healing via induction and infrared heating. Constr Build Mater, 2016, 126: 957-966

57 Liu Q, Schlangen E, van de Ven M, et al. Healing of porous asphalt concrete via induction heating. Road Mater Pavement Des, 2010, 11: 527-542 
58 Liu Q, García Á, Schlangen E, et al. Induction healing of asphalt mastic and porous asphalt concrete. Constr Build Mater, 2011, 25: 3746-3752

59 García A, Bueno M, Norambuena-Contreras J, et al. Induction healing of dense asphalt concrete. Constr Build Mater, 2013, 49: 1-7

60 Zhao H, Zhong S, Zhu X, et al. High-efficiency heating characteristics of ferrite-filled asphalt-based composites under microwave irradiation. J Mater Civil Eng, 2017, 29: 04017007

61 Zhu X, Cai Y, Zhong S, et al. Self-healing efficiency of ferrite-filled asphalt mixture after microwave irradiation. Constr Build Mater, 2017, 141: $12-22$

62 Barbero E J, Greco F, Lonetti P. Continuum damage-healing mechanics with application to self-healing composites. Int J Damage Mech, 2005, 14: 51-81

63 Darabi M K, Abu Al-Rub R K, Little D N. A continuum damage mechanics framework for modeling micro-damage healing. Int J Solids Struct, 2012, 49: 492-513

64 Barenblatt G I. The mathematical theory of equilibrium cracks in brittle fracture. Adv Appl Mech, 1962, 7: 55-129

65 Dugdale D S. Yielding of steel sheets containing slits. J Mech Phys Solids, 1960, 8: 100-104

66 Klein P A, Foulk J W, Chen E P, et al. Physics-based modeling of brittle fracture: Cohesive formulations and the application of meshfree methods. Theor Appl Fract Mech, 2001, 37: 99-166

67 Abu Al-Rub R K, Darabi M K, Little D N, et al. A micro-damage healing model that improves prediction of fatigue life in asphalt mixes. Int J Eng Sci, 2010, 48: 966-990

68 Voyiadjis G Z, Shojaei A, Li G, et al. A theory of anisotropic healing and damage mechanics of materials. Pro Roy Soc, 2012, 468: 163-183

69 Esgandani G A, El-Zein A. Thermodynamic based model for coupled elastoplastic damage-healing behaviour of unsaturated geomaterials. Mech Mater, 2020, 145: 103395

70 Voyiadjis G Z, Shojaei A, Li G. A thermodynamic consistent damage and healing model for self healing materials. Int J Plast, 2011, 27: 1025-1044

71 Oucif C, Voyiadjis G Z, Rabczuk T. Modeling of damage-healing and nonlinear self-healing concrete behavior: Application to coupled and uncoupled self-healing mechanisms. Theor Appl Fract Mech, 2018, 96: 216-230

72 Shahsavari H, Baghani M, Naghdabadi R, et al. A thermodynamically consistent viscoelastic-viscoplastic constitutive model for self-healing materials. J Intell Mater Syst Struct, 2018, 29: 1065-1080

73 Darabi M K, Abu Al-Rub R K, Masad E A, et al. A thermo-viscoelastic-viscoplastic-viscodamage constitutive model for asphaltic materials. Int J Solids Struct, 2011, 48: 191-207

74 Darabi M K, Abu Al-Rub R K, Masad E A, et al. Constitutive modeling of fatigue damage response of asphalt concrete materials with consideration of micro-damage healing. Int J Solids Struct, 2013, 50: 2901-2913

75 Alsheghri A A, Abu Al-Rub R K. Thermodynamic-based cohesive zone healing model for self-healing materials. Mech Res Commun, 2015, 70: 102-113

76 Schapery R A. On the mechanics of crack closing and bonding in linear viscoelastic media. Int J Fract, 1989, 39: 163-189

77 Perzyna P. Thermodynamic theory of viscoplastcity. Adv Appl Mech, 1971, 11: 313-354

78 Lai J, Bakker A. 3-D schapery representation for non-linear viscoelasticity and finite element implementation. Comput Mech, 1996, 18: 182-191

79 Oucif C, Voyiadjis G Z, Kattan P I, et al. Investigation of the super healing theory in continuum damage and healing mechanics. Int J Damage Mech, 2019, 28: 896-917

80 Bhasin A, Bommavaram R, Greenfield M L, et al. Use of molecular dynamics to investigate self-healing mechanisms in asphalt binders. J Mater Civil Eng, 2011, 23: 485-492

81 Jennings P W, Pribanic J A, Desando M A, et al. Binder characterization and evaluation by nuclear magnetic resonance spectroscopy. In: Strategic Highway Research Program. Washington: National Research Council, 1993, SHRP-A-335

82 Shen S, Lu X, Liu L, et al. Investigation of the influence of crack width on healing properties of asphalt binders at multi-scale levels. Constr Build Mater, 2016, 126: 197-205

$83 \mathrm{Xu} \mathrm{G}$, Wang H. Molecular dynamics study of oxidative aging effect on asphalt binder properties. Fuel, 2017, 188: 1-10

84 Sun D, Lin T, Zhu X, et al. Indices for self-healing performance assessments based on molecular dynamics simulation of asphalt binders. Comput Mater Sci, 2016, 114: 86-93

85 Gilford Iii J, Hassan M M, Rupnow T, et al. Dicyclopentadiene and sodium silicate microencapsulation for self-healing of concrete. J Mater Civil Eng, 2014, 26: 886-896

86 Hilloulin B, Van Tittelboom K, Gruyaert E, et al. Design of polymeric capsules for self-healing concrete. Cement Concrete Compos, 2015, 55: 298307

87 Souradeep G, Kua H W. Encapsulation technology and techniques in self-healing concrete. J Mater Civil Eng, 2016, 28: 04016165

88 Pang J, Bond I. 'Bleeding composites'-damage detection and self-repair using a biomimetic approach. Compos Part A-Appl Sci Manuf, 2005, 36: 183-188 
89 Zemskov S V, Jonkers H M, Vermolen F J. Two analytical models for the probability characteristics of a crack hitting encapsulated particles: Application to self-healing materials. Comput Mater Sci, 2011, 50: 3323-3333

90 Gilabert F A, Garoz D, Paepegem W V. Macro- and micro-modeling of crack propagation in encapsulation-based self-healing materials: Application of XFEM and cohesive surface techniques. Mater Des, 2017, 130: 459-478

91 Xue C, Li W, Li J, et al. Numerical investigation on interface crack initiation and propagation behaviour of self-healing cementitious materials. Cement Concrete Res, 2019, 122: 1-16

92 Mauludin L M, Oucif C. Interaction between matrix crack and circular capsule under uniaxial tension in encapsulation-based self-healing concrete. Underground Space, 2018, 3: 181-189

93 Shojaei A, Sharafi S, Li G. A multiscale theory of self-crack-healing with solid healing agent assisted by shape memory effect. Mech Mater, 2015, 81: $25-40$

94 Ponnusami S A, Krishnasamy J, Turteltaub S, et al. A cohesive-zone crack healing model for self-healing materials. Int J Solids Struct, 2018, 134: 249-263

95 Alsheghri A A, Abu Al-Rub R K. Finite element implementation and application of a cohesive zone damage-healing model for self-healing materials. Eng Fract Mech, 2016, 163: 1-22 


\title{
Application of self-healing engineering materials: Mechanical problems and research progress
}

\author{
Xingyi Zhu ${ }^{1}$, Chenghong $\mathrm{Lu}^{1}$, Ziwei Dai ${ }^{1} \& \mathrm{Feng} \mathrm{Li}^{2 *}$ \\ ${ }^{1}$ Key Laboratory of Road and Traffic Engineering of the Ministry of Education, Tongji University, Shanghai 201804, China; \\ ${ }^{2}$ School of Transportation Science and Engineering, Beihang University, Beijing 102206, China \\ * Corresponding author, E-mail: lifeng98@buaa.edu.cn
}

In order to meet the needs of future infrastructure and industrial construction, namely building new advanced infrastructures with intelligent capabilities, the optimization of the structure and material composition design in traditional civil engineering has aroused general interest in both industrial and academic fields. The damage resistance of engineering structure has a direct impact on the social and economic cost to a country. To considerably reduce the maintenance cost and improve the service life of engineering structures, a feasible scheme is to build a life-like system with the capability of selfhealing. In recent years, techniques like microcapsule self-healing, electrodeposition self-healing, induction heating selfhealing and microbial self-healing have been widely used in civil engineering and road engineering, which are expected to improve the durability and stability of engineering structures, thus prolonging their service life to the maximum extent. However, the improvement of the performance of self-healing engineering materials and the accurate prediction of the crack propagating trajectory and the service life of the materials require a better understanding of the self-healing mechanism. In this review, the development and the advancement of the application of self-healing materials in civil engineering are firstly summarized. The self-healing mechanisms of cement concrete and asphalt, the two most used materials in civil engineering, are concluded, respectively. However, due to the low efficiency of the materials' natural selfhealing capability, some enhancement technologies are proposed and applied to improve the self-healing performance of those engineering materials, which are summarized in the later introduction. In the second part, the mechanical analysis for the explanation and prediction of the self-healing behaviors is introduced in detail based on damage and fracture mechanics. Besides, there are still some mechanical problems that remain unsolved, such as decoupling of elastic effect and self-healing effect on macro performance recovery and establishing a fatigue model considering damage evolution, crack propagation and self-healing effect, which are also summarized and analyzed. In the third part, the existing constitutive models considering self-healing effects and the relevant numerical algorithms are reviewed. Based on the three basic hypotheses, namely the strain equivalent hypothesis, the strain energy equivalent hypothesis and the power equivalent hypothesis, the effective configuration considering damage-healing effect is described. Additionally, relatively detailed review is given on the numerical algorithms considering self-healing mechanism with the aid of molecular dynamics (MD) and finite element method (FEM). Finally, the unsolved problems and challenges are proposed for further researches, where the coupling effects of the internal and external factors on the damage-fracture-healing process will be studied from a mechanical point of view. Therefore, the deep understanding of the healing and damage behavior of materials needs sufficient multi-scale analysis involving nano-scale, micro-scale, meso-scale and macro-scale. To sum up, self-healing materials play an important role in improving the durability and reliability of engineering structures. Therefore, the improvement of the self-healing efficiency is the key point to realize the wide and high efficient application of self-healing materials in civil engineering, which needs not only an essential understanding of the mechanical mechanisms but also fast numerical algorithms to accurately evaluate and predict the self-healing behavior.

self-healing, constitutive model, continuum damage healing mechanics, multi-scale, numerical simulation

doi: 10.1360/TB-2020-1082 